\begin{tabular}{|c|l|}
\hline Title & EQUIV A LENCE OF BMO-TYPE NORMS WITH APPLICA TIONS TO THE HEAT AND STOKES SEMIGROUPS \\
\hline Author(s) & BOLKART, MA RTIN; GIGA, Y OSHIKAZU; Suzuki, Takuya; TSUTSUI, Y OHEI \\
\hline Citation & Hokkaido University Preprint Series in Mathematics, 1090, 1-28 \\
\hline Issue Date & 2016-5-12 \\
\hline DOI & 10.14943/84234 \\
\hline Doc URL & http://hdl.handle.net/2115/69894 \\
\hline Type & bulletin (article) \\
\hline File Information & pre1090.pdf \\
\hline
\end{tabular}

Instructions for use 


\title{
EQUIVALENCE OF $B M O$-TYPE NORMS WITH APPLICATIONS TO THE HEAT AND STOKES SEMIGROUPS
}

\author{
MARTIN BOLKART, YOSHIKAZU GIGA, TAKUYA SUZUKI, \\ AND YOHEI TSUTSUI
}

\begin{abstract}
We introduce various spaces of functions of bounded mean oscillations $(B M O)$ defined in a domain by taking into account the behavior of functions near the boundary. Then we establish several equivalences of these spaces. Moreover, we compare our space with a $B M O$ space introduced by Miyachi. As an application we prove that the heat and the Stokes semigroup are analytic in such a type of spaces.
\end{abstract}

\section{INTRODUCTION}

In this article, we discuss equivalences of BMO-type norms in domains. Since we will consider the behavior of functions near the boundary, our $B M O$ norms consist of an interior and a boundary part. The reason we are interested in such problems is to prove analyticity of the heat and Stokes semigroup in domains.

The space $B M O\left(\mathbb{R}^{n}\right)$ has previously been introduced by the seminal paper of John and Nirenberg [22]. Fefferman [11] showed that $B M O\left(\mathbb{R}^{n}\right)$ is the dual of the Hardy space $\mathcal{H}^{1}\left(\mathbb{R}^{n}\right)$ and a decomposition of functions in $B M O\left(\mathbb{R}^{n}\right)$ in terms of Riesz transforms. A constructive proof of the last result was given by Uchiyama [39]. The theory of $B M O\left(\mathbb{R}^{n}\right)$ was developed in the remarkable paper of Fefferman and Stein [12]. BMO spaces play important roles in harmonic analysis and PDEs, as a substitute of $L^{\infty}$. Several operators in these fields are not bounded on $L^{\infty}$, but from $L^{\infty}$ to $B M O$. Moreover, the real and complex interpolation theories work with $B M O$. For example, $L^{p}$ coincides with interpolation spaces with $B M O$ space, [19], [21].

We already know ways to characterize functions in $B M O\left(\mathbb{R}^{n}\right)$. For instance, Carleson measures ([6], [12], [37]), $A_{p}$-weights ([13]) and LittlewoodPaley decomposition ([38]). The space $B M O\left(\mathbb{R}^{n}\right)$ appears in several problems in harmonic analysis; paraproduct [5], commutator of singular integrals

2010 Mathematics Subject Classification. 30H35; 35K05, 47D03, 76D07.

Key words and phrases. BMO, heat equation, Stokes equations, analytic semigroups.

This work was partly supported by the Japan Society for the Promotion of Science (JSPS) and the German Research Foundation through the Japanese-German Graduate Externship and International Research Training Group 1529 on Mathematical Fluid Dynamics. The second author is partly supported by JSPS through grants Kiban S (No. 26220702), Kiban A (No. 23244015) and Houga (No. 25610025). The third author is partly supported by the Program for Leading Graduate Schools, JSPS and MEXT as the Leading Graduate Course for Frontiers of Mathematical Sciences and Physics (FMSP). The fourth author is partly supported by JSPS, Grant-in-Aid for Young Scientists (B) (No. 15K20919) and Grant-in-Aid for Scientific Research (B) (No. 23340034). 
[9], $T(1)$ theorem [10], and in PDEs, especially in fluid dynamics; wellposedness for incompressible Navier-Stokes equations on the whole space [24] and a blow up criterion for the same equation [26].

If one considers the space $B M O$ in a domain $\Omega$, the situation is less clear compared with the case of the whole space $\mathbb{R}^{n}$. To discuss possible definitions of $B M O$ in a domain, we will define various types of $B M O$-type (semi)norms. Sometimes we have to be careful about the behavior near the boundary $\partial \Omega$. For this purpose we define for $f \in L_{l o c}^{1}(\Omega), \nu \in(0, \infty]$ and $p \in[1, \infty)$ the seminorm

$$
[f]_{b^{\nu} p}:=\sup \left\{\left(r^{-n} \int_{\Omega \cap B_{r}(x)}|f(y)|^{p} d y\right)^{1 / p}: x \in \partial \Omega, 0<r<\nu\right\},
$$

where $B_{r}(x)$ denotes the closed ball of radius $r$ centered at $x$ (cf. Remark $6)$. For $\mu \in(0, \infty]$ we define

$$
[f]_{B M O^{\mu} p}:=\sup \left\{\left(\frac{1}{\left|B_{r}(x)\right|} \int_{B_{r}(x)}\left|f(y)-f_{B_{r}(x)}\right|^{p} d y\right)^{1 / p}: B_{r}(x) \subset \Omega, r<\mu\right\},
$$

where for any ball $B \subset \mathbb{R}^{n}$,

$$
f_{B}:=\frac{1}{|B|} \int_{B} f(y) d y .
$$

Then our $B M O$ space is defined by the norm

$$
\|f\|_{B M O_{b}^{\mu, \nu} p}:=[f]_{B M O^{\mu} p}+[f]_{b^{\nu} p} .
$$

If one replaces balls by cubes in the above definition for $[\cdot]_{B M O^{\mu}}$ one gets an equivalent seminorm. For a proof of this fact for general domains we refer to [35]. We then let $B M O_{b}^{\mu, \nu}(\Omega)$ be the space of all functions $f \in$ $L_{\text {loc }}^{1}(\Omega)$ satisfying $\|f\|_{B M O_{b}^{\mu, \nu}}<\infty$. Furthermore, the space $V M O_{b, 0}^{\mu, \nu}(\Omega)$ is defined as the closure of $C_{c}^{\infty}(\Omega)$ in $B M O_{b}^{\mu, \nu}(\Omega)$ and the solenoidal space $V M O_{b, 0, \sigma}^{\mu, \nu}(\Omega)$ is defined as the closure of $C_{c, \sigma}^{\infty}(\Omega)$ in $B M O_{b}^{\mu, \nu}(\Omega)$. Similarly, $C_{0}(\Omega)$ and $C_{0, \sigma}(\Omega)$ are defined as the $L^{\infty}(\Omega)$-closure of $C_{c}^{\infty}(\Omega)$ and $C_{c, \sigma}^{\infty}(\Omega)$, respectively.

There exists a similar definition of the $B M O_{b}$-norm that was used by A. Miyachi in [31]. We generalize his norm to $p \in[1, \infty)$ by

$$
\begin{aligned}
& {[f]_{B M O^{M} p}:=\sup \left\{\left(\frac{1}{\left|B_{r}(x)\right|} \int_{B_{r}(x)}\left|f-f_{B_{r}(x)}\right|^{p} d y\right)^{\frac{1}{p}}: B_{2 r}(x) \subset \Omega\right\}} \\
& {[f]_{b^{M} p}:=\sup \left\{\left(\frac{1}{\left|B_{r}(x)\right|} \int_{B_{r}(x)}|f|^{p} d y\right)^{\frac{1}{p}}: B_{2 r}(x) \subset \Omega \text { and } B_{5 r}(x) \cap \Omega^{c} \neq \emptyset\right\}}
\end{aligned}
$$

and

$$
\|f\|_{B M O_{b}^{M} p}:=[f]_{B M O^{M} p}+[f]_{b^{M} p} .
$$

For the case $p=1$, we omit $p$ in the definitions above. 
Main results in this paper consist of three types of equivalences for $B M O_{b}^{\mu, \nu} p$ norms:

[I] equivalence for any $\mu, \nu \in(0, \infty]$ (Theorems $5,6,7,8)$

[II] equivalence for the power $p \in[1, \infty)$ (Theorems 13,14 )

[III] equivalence to $B M O_{b}^{M} p$ (Theorems 9,10 )

The main ingredients of the proofs of [I] and [III] are Jones' extension theorem (Theorem 1) and an $L^{1}$-growth estimate for $B M O$ functions (Theorem 3 ). The proof of [II] makes use of $L^{1}-B M O$ interpolation in $\mathbb{R}^{n}$ (Lemma 5) and careful investigation of Jones' construction for his extension operator.

As it is mentioned above, some of our results make use of extension arguments. Although for any domain the extension of $L^{\infty}$ functions by 0 does not cause problems, it is an interesting problem for $B M O$ functions on domains. Jones [23] gave a sufficient condition on domains for the existence of a bounded extension operator. Since his operator is needed in our aims, we recall its construction in the next section. But for some domains, the zero extension of $B M O$ functions is useful, see Lemma 4. One can see that layer domains do not fulfill the Jones condition and have no extension operator, see Remark 1.

As the first application we study the analyticity of the heat semigroup, the solution operator $H: u_{0} \mapsto H(t) u_{0}=u(\cdot, t)$, where $u$ is the solution to

$$
\begin{aligned}
& u_{t}-\Delta u=0 \quad \text { in } \Omega \times(0, T) \text {, } \\
& u=0 \quad \text { on } \partial \Omega \times(0, T), \\
& \left.u\right|_{t=0}=0 \quad \text { on } \Omega
\end{aligned}
$$

in $B M O$-type spaces when $\Omega$ is a domain in $\mathbb{R}^{n}$. If $\Omega$ is $\mathbb{R}^{n}$, the whole space, a key estimate

$$
\sup _{t>0}\left([u(t)]_{B M O^{\infty}}+t\left\|u_{t}(t)\right\|_{\infty}\right) \leq C\left[u_{0}\right]_{B M O^{\infty}}
$$

is easily obtained from a corresponding estimate in Hardy spaces and a duality argument; see Theorem 15 where spatial derivatives up to second order are also controlled. Note that instead of the $B M O$-type norm the $L^{\infty}$ norm of the time derivative $\left\|u_{t}\right\|_{\infty}$ is controlled and this gives a regularizing effect from $B M O$ to $L^{\infty}$. If $\Omega$ is the half space $\mathbb{R}_{+}^{n}$, then an estimate similar to (1.1) is obtained by replacing $B M O^{\infty}$ by $B M O_{b}^{\infty} \cdot \infty$, i.e.,

$$
\sup _{t>0}\left([u(t)]_{B M O_{b}^{\infty, \infty}}+t\left\|u_{t}(t)\right\|_{\infty}\right) \leq C\left[u_{0}\right]_{B M O_{b}^{\infty, \infty}}
$$

This is obtained by an odd extension and (1.1); see Theorem 16 which seems to be not included in the literature. In both estimates $C$ is a positive constant depending only on the space dimension $n$. From (1.2) we are able to prove that $H(t)$ is a (non $C_{0}$ ) bounded analytic semigroup in $B M O_{b}^{\infty, \infty}$ and a $C_{0}$ bounded analytic semigroup in $V M O_{b, 0}^{\infty, \infty}$ when $\Omega$ is the half space. 
For a general uniformly $C^{3}$-domain $\Omega$ we shall establish a similar estimate but local-in-time of the form

$$
\begin{array}{r}
\sup _{0<t<T_{0}}\left(\|u(t)\|_{B M O_{b}^{\mu, \nu}}+t^{1 / 2}\|\nabla u(t)\|_{\infty}+t\left\|\nabla^{2} u(t)\right\|_{\infty}+t\left\|u_{t}(t)\right\|_{\infty}\right) \\
\leq C\left\|u_{0}\right\|_{B M O_{b}^{\mu, \nu}}
\end{array}
$$

with some constants $C$ and $T_{0}$ independent of $u_{0} \in V M O_{b}^{\mu, \nu}$ when $\mu \in(0, \infty]$ and $\nu$ is smaller than the reach of $\partial \Omega$ (Theorem 18). The regularity part (estimate for $\nabla u, \nabla^{2} u, u_{t}$ ) is obtained by a blow-up argument similar to the one developed in [2] while the estimate for $u$ is obtained by an argument similar to the one in [3]; both papers discuss the Stokes semigroup.

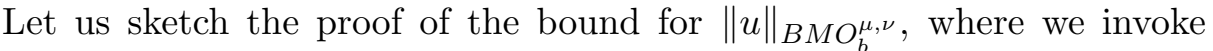
equivalence of $B M O_{b}^{\mu, \nu} p$ for $p=1$ and $p=2$. The proof consists of four steps. First, we derive a pointwise mean value estimate of a solution with respect to the time variables (Lemma $9(1)$ ). This is obtained by the $L^{\infty}$ $B M O$ type estimate for the gradient. Second and third, we estimate the $B M O^{\mu} 2$ seminorm in two ways by using the $L^{\infty}-B M O$ type estimates and the Poincaré inequality (Lemma $9(2),(3)$ ). Fourth, we estimate the $b^{\nu} 2$ seminorm by a similar argument (Lemma $9(4)$ ). Here we invoke the equivalence of $B M O_{b}^{\mu, \nu}$ and $B M O_{b}^{\mu, \nu} p$. Note that an estimate similar to (1.3) holds for $V M O_{b, 0}^{M}(\Omega)$. Thus we are able to conclude that $H$ is a $C_{0}$-analytic semigroup in $V M O_{b, 0}^{M}(\Omega)$.

As the second application we study the analyticity of the Stokes semigroup $S$, the solution operator of the Stokes equations, in $V M O_{b, 0, \sigma}^{\mu, \nu}(\Omega)$ when $\Omega$ is an uniformly $C^{3}$ and admissible. Such a result was obtained for sufficiently small $\nu$ in [3]. By the equivalence result (Theorem 5) one can extend this result to general $\mu, \nu \in(0, \infty]$ in bounded domains. Furthermore, one is able to prove that $S$ is bounded in $V M O_{b, 0, \sigma}^{\mu, \nu}(\Omega)$ for $t>0$ when $\Omega$ is bounded. For analyticity in $V M O_{b, 0, \sigma}^{\mu, \nu}$ one is able to prove that $S$ is analytic if $\Omega$ is an admissible Lipschitz half-space with uniformly $C^{3}$-boundary including the case $\mu=\nu=\infty$, which is not included in [3]. This analyticity results also extends to $V M O_{b, 0, \sigma}^{M}$.

Let us review literature concerning $B M O$ type estimates of the heat equation in $\mathbb{R}^{n}$. A. Carpio [7] and the second author, S. Matsui, Y. Shimizu [16] established $\mathcal{H}^{1}-L^{1}$ estimates which by duality imply $L^{\infty}-B M O$ gradient estimates:

$$
t^{\frac{1}{2}}\left\|\nabla G_{t} * u_{0}\right\|_{L^{\infty}\left(\mathbb{R}^{n}\right)} \leq C\left[u_{0}\right]_{B M O^{\infty}\left(\mathbb{R}^{n}\right)}
$$

where $G_{t}$ denotes the Gaussian kernel and $*$ the convolution. We remark that $\left\|G_{t} * u_{0}\right\|_{L^{\infty}\left(\mathbb{R}^{n}\right)}$ is not bounded by $\left[u_{0}\right]_{B M O\left(\mathbb{R}^{n}\right)}$ which can be observed by taking $u_{0}$ constant. Moreover, this $L^{\infty}-B M O$ estimate for the gradient cannot be generalized to the case when a domain has nonempty boundary under the Dirichlet condition since $u$ may not be spatially constant even if $u_{0}$ is a constant. In [25] and also in [34, Lemma 14.4.1], $B M O\left(\mathbb{R}^{n}\right)$ estimates and $L^{\infty}-B M O$ estimates for $e^{t \Delta} u_{0}, \nabla e^{t \Delta} u_{0}, \nabla^{2} e^{t \Delta} u_{0}$ were established:

$$
\begin{aligned}
{\left[\nabla^{k} e^{t \Delta} u_{0}\right]_{B M O^{\infty}\left(\mathbb{R}^{n}\right)} } & \leq C t^{-\frac{k}{2}}\left[u_{0}\right]_{B M O^{\infty}\left(\mathbb{R}^{n}\right)} \text { for } k=0,1,2, \\
\left\|\nabla^{k} e^{t \Delta} u_{0}\right\|_{L^{\infty}\left(\mathbb{R}^{n}\right)} & \leq C t^{-\frac{k}{2}}\left[u_{0}\right]_{B M O^{\infty}\left(\mathbb{R}^{n}\right)} \text { for } k=1,2 .
\end{aligned}
$$


The $B M O^{\infty}\left(\mathbb{R}^{n}\right)$ estimates are obtained by $\mathcal{H}^{p}-\mathcal{H}^{q}$ estimates, see [20], [32], [33], and a duality argument. $L^{\infty}-B M O$ estimates for the gradients are also obtained by a duality argument.

This paper is organized as follows. In Section 2 we recall several properties of Jones' extension. In Section 3 we discuss equivalences of different $B M O_{b}^{\mu, \nu}$ by changing $\mu$ and $\nu$ for various domains including some domains which do not allow Jones' extension. We conclude this section by discussing the equivalence of $B M O_{b}^{\mu, \nu} p$ when $p$ is different. In Section 4 we discuss analyticity of the heat semigroup in $B M O$ type spaces and in Section 5 we discuss the analyticity of the Stokes semigroup in $B M O$ type spaces.

\section{JONES' EXTENSION THEOREM}

We will need to consider certain classes of domains in order to compare different $B M O$-type norms or to prove embeddings from $B M O$-type spaces to $L^{p}$. For the existence of an extension operator on $B M O^{\infty}(\Omega)$ we will need the notion of a uniform domain. In some cases we will also need $C^{2}$ boundary to get control over the ratio $\left|B_{r}\left(x_{0}\right)\right| /\left|B_{r}\left(x_{0}\right) \cap \Omega\right|$ for small $r$ and $x_{0} \in \partial \Omega$. Both properties are crucial in several proofs.

Lemma 1. Let $\Omega$ be a uniformly $C^{2}$-domain. Then there exists a constant $R>0$ depending only on $C^{2}$-regularity of $\Omega$ such that there is a projection $P_{\partial \Omega}:\left\{x \in \mathbb{R}^{n}: \operatorname{dist}(x, \partial \Omega)<R\right\} \rightarrow \partial \Omega$ with $P_{\partial \Omega} x-x=\operatorname{dist}(x, \partial \Omega) \mathbf{n}$, where $\mathbf{n}$ is the exterior normal at $\partial \Omega$ in $P_{\partial \Omega} x$ if $x \in \Omega$ and the interior normal of $\partial \Omega$ at $P_{\partial \Omega} x$ if $x \notin \Omega$. Note that $P_{\partial \Omega} x$ is uniquely determined if $d(x, \partial \Omega)<R$.

Proof. For a proof see [17, appendix] and [28, §4.4].

We define then for a $C^{2}$-domain the reach of $\Omega$ denoted by $R^{*}>0$ to be the supremum of all $R$ as in the above Lemma. The reach of $\Omega$ then depends only on $C^{2}$-regularity of $\Omega$.

For several equivalence proofs we will need an extension theorem for $B M O$ functions on domains that is due to P. W. Jones ([23]). Since the construction of this extension will be important for our needs, we will give a sketch of this construction. In order to do so we need to define the dyadic Whitney decomposition of a set $A$.

For a set $A \subset \mathbb{R}^{n}$ let $\mathcal{A}=\left\{Q_{j}\right\}_{j \in \mathbb{N}}$ be a set of dyadic closed cubes with side length $\ell\left(Q_{j}\right)$ contained in $A$ such that

(1) $A=\cup_{j} Q_{j}$

(2) $\stackrel{\circ}{Q}_{j} \cap \dot{Q}_{k}=\emptyset$ if $j \neq k$

(3) $1 \leq \frac{d\left(Q_{j}, \mathbb{R}^{n} \backslash A\right)}{\ell\left(Q_{j}\right)} \leq 4 \sqrt{n}(j \in \mathbb{N})$

(4) $\frac{1}{4} \leq \frac{\ell\left(Q_{k}\right)}{\ell\left(Q_{j}\right)} \leq 4$ if $Q_{j} \cap Q_{k} \neq \emptyset$.

Then $\mathcal{A}$ will be called a dyadic Whitney decomposition of $A$. For the existence of the Whitney decomposition for open sets we refer to [36, Chapter VI, Theorem 1].

We define two different distance functions on the Whitney decomposition. For $Q_{j}, Q_{k} \in \mathcal{A}$ we call $Q_{j}=Q(0) \rightarrow Q(1) \rightarrow Q(2) \ldots \rightarrow Q(m)=Q_{k}$ a Whitney chain of length $m$ connecting $Q_{j}$ and $Q_{k}$ if $Q(l) \in \mathcal{A}$ for all $0 \leq$ $\ell \leq m$ and $Q(l) \cap Q(l+1) \neq \emptyset$ for each $0 \leq l \leq m-1$. The distance function 
$d_{1}\left(Q_{j}, Q_{k}\right)$ will then be defined as the length of the shortest Whitney chain connecting $Q_{j}$ and $Q_{k}$.

For $Q_{j}, Q_{k} \in \mathcal{A}$ we define the second distance function as

$$
d_{2}\left(Q_{j}, Q_{k}\right):=\log \left|\frac{\ell\left(Q_{j}\right)}{\ell\left(Q_{k}\right)}\right|+\log \left|\frac{d\left(Q_{j}, Q_{k}\right)}{\ell\left(Q_{j}\right)+\ell\left(Q_{k}\right)}+2\right|,
$$

where $d$ denotes the Euclidean distance between the cubes. Note that $d_{1}$ and $d_{2}$ are scale invariant.

A domain $A \subset \mathbb{R}^{n}$ will be called a uniform domain if there is some $K>0$ such that

$$
d_{1}\left(Q_{j}, Q_{k}\right) \leq K d_{2}\left(Q_{j}, Q_{k}\right)
$$

for all $Q_{j}, Q_{k} \in \mathcal{A}$ and some dyadic Whitney decomposition $\mathcal{A}$. The name uniform is due to the following equivalent definition of this class of domains ([14]). A domain $\Omega$ is uniform if there exist constants $a, b>0$ such that for all $x, y \in \Omega$ there is a rectifiable curve $\gamma \subset \Omega$ of length $s(\gamma) \leq a|x-y|$ with $\min \{s(\gamma(x, z)), s(\gamma(y, z))\} \leq b \operatorname{dist}(z, \partial \Omega)$, where $\gamma(x, z)$ denotes the part of $\gamma$ between $x$ and $z$. Bounded Lipschitz domains are examples of uniform domains.

We are now able to formulate the extension theorem for $B M O$ functions.

Theorem 1. Let $A \subset \mathbb{R}^{n}$ be a uniform domain. Then there is a constant $C(K)>0$ such that for each $f \in B M O^{\infty}(A)$ there is an extension $\bar{f} \in$ $B M O^{\infty}\left(\mathbb{R}^{n}\right)$ such that

$$
[\bar{f}]_{B M O \infty\left(\mathbb{R}^{n}\right)} \leq C(K)[f]_{B M O^{\infty}(A)},
$$

where $K$ is the constant in (2.1). In particular, the theorem holds for bounded Lipschitz domains with a constant only depending on the Lipschitz regularity of $A$. If there exists such an extension for all $f \in B M O^{\infty}(A)$, then $\Omega$ is uniform.

Proof. The theorem is due to [23].

We will repeat the explicit construction of $\bar{f}$. Let $A^{c}$ be the complement of $A$ and $\mathcal{A}^{\prime}$ be the Whitney decomposition of its interior. Choose for every $Q_{j}^{\prime} \in \mathcal{A}^{\prime}$ a corresponding $Q_{j} \in \mathcal{A}$ in the following way. If there are cubes $Q_{j} \in \mathcal{A}$ which satisfy $\ell\left(Q_{j}\right) \geq \ell\left(Q_{j}^{\prime}\right)$ then choose the nearest cube $Q_{j} \in \mathcal{A}$ satisfying $\ell\left(Q_{j}\right) \geq \ell\left(Q_{j}^{\prime}\right)$. For all other cubes choose some largest cube $Q_{0} \in \mathcal{A}$ and let $Q_{0}$ be the cube corresponing to all $Q_{j}^{\prime} \in \mathcal{A}^{\prime}$ for which there are no cubes in $Q_{j} \in \mathcal{A}$ satisfying $\ell\left(Q_{j}\right) \geq \ell\left(Q_{j}^{\prime}\right)$. The second case appears for example if $A$ is a bounded domain. Then $\bar{f}$ is defined as

$$
\bar{f}(x):=\left\{\begin{array}{ll}
f(x) & : x \in A \\
f_{Q_{j}} & : x \in Q_{j}^{\prime}
\end{array},,\right.
$$

where $Q_{j} \in A$ is the cube corresponding to $Q_{j}^{\prime}$. Since by [23, Corollary 2.9] $|\partial \Omega|=0$ for uniform domains, we can ignore the boundary of $\Omega$ in the construction.

Furthermore, we will need the following lemma (cf. [23, Lemma 2.10]). 
Lemma 2. Let $A \subset \mathbb{R}^{n}$ be a uniform domain, $\mathcal{A}$ and $\mathcal{A}^{\prime}$ be the Whitney decomposition of $A$ and $A^{c}$ respectively and let $Q_{j}^{\prime} \in \mathcal{A}^{\prime}$. If there exists a cube $Q \in \mathcal{A}$ with $\ell(Q) \geq \ell\left(Q_{j}^{\prime}\right)$, then

$$
d\left(Q_{j}, Q_{j}^{\prime}\right) \leq 65 K^{2} \ell\left(Q_{j}^{\prime}\right) \leq 65 K^{2} \ell\left(Q_{j}\right)
$$

with $K$ the number obtained in condition (2.1) and $Q_{j}$ the cube corresponding to $Q_{j}^{\prime}$.

Remark 1. Domains of the form $\Omega=\mathbb{R}^{k} \times G$ with $1 \leq k \leq n-1$ and bounded $G \subset \mathbb{R}^{n-k}$ are examples of domains which are not uniform. We will show that for such domains there is no Jones' extension. Let $f(x)=x_{1}$, then for every cube $Q$ in $\Omega$

$$
\begin{aligned}
\frac{1}{|Q|} \int_{Q}\left|f-f_{Q}\right| d y & =\frac{\ell(Q)^{n-1}}{|Q|} \int_{-\ell(Q) / 2}^{\ell(Q) / 2}\left|x_{1}\right| d x_{1} \\
& =\frac{1}{4} \ell(Q) .
\end{aligned}
$$

Thus $f \in B M O^{\infty}(\Omega)$ because the cubes in $\Omega$ have side length of at most $\operatorname{diam}(G)$. This function cannot be extended to a function $\bar{f} \in B M O^{\infty}\left(\mathbb{R}^{n}\right)$ since otherwise $B M O^{\infty}\left(\mathbb{R}^{n}\right)$ would contain functions of linear growth.

3. EMbeddings AND EQUIVALENCES OF BMO-TYPE NORMS

Theorem 2. Let $\Omega \subset \mathbb{R}^{n}$ be a domain and $\mu, \nu \in(0, \infty]$. Then the embeddings

$$
\begin{aligned}
L^{\infty}(\Omega) & \hookrightarrow B M O_{b}^{\mu, \nu}(\Omega), \\
C_{0}(\Omega) & \hookrightarrow V M O_{b, 0}^{\mu, \nu}(\Omega), \\
C_{0, \sigma}(\Omega) & \hookrightarrow V M O_{b, 0, \sigma}^{\mu, \nu}(\Omega)
\end{aligned}
$$

hold with an embedding constant depending only on $n$, i.e., independent of $\Omega, \mu$ and $\nu$.

Proof. It follows from the definition of the norm that $\|f\|_{B M O_{b}^{\mu, \nu}} \leq(2+$ $\left.\omega_{n}\right)\|f\|_{\infty}$, where $\omega_{n}=\left|B_{1}(0)\right|$ is the measure of the unit ball in $\mathbb{R}^{n}$.

Remark 2. It follows from the definition that for $0<\mu_{1} \leq \mu_{2} \leq \infty$ and $0<\nu_{1} \leq \nu_{2} \leq \infty$ the estimates

$$
[f]_{B M O^{\mu_{1}}} \leq[f]_{B M O^{\mu_{2}}}, \quad[f]_{b^{\nu_{1}}} \leq[f]_{b^{\nu_{2}}}
$$

and the embedding

$$
B M O_{b}^{\mu_{1}, \nu_{1}}(\Omega) \hookrightarrow B M O_{b}^{\mu_{2}, \nu_{2}}(\Omega)
$$

hold.

Theorem 3. Let $\mu \in(0, \infty]$ and $\Omega \subset \mathbb{R}^{n}$ be a domain. Then for all $f \in$ $B M O^{\mu}(\Omega), a>1, r>0, x_{1}, x_{2} \in \Omega$ with $B_{r}\left(x_{1}\right) \subset B_{a r}\left(x_{2}\right) \subset \Omega$ and ar $<\mu$ the inequality

$$
\|f\|_{L^{1}\left(B_{a r}\left(x_{2}\right)\right)} \leq\left|B_{a r}\left(x_{2}\right)\right|\left(1+a^{n}\right)[f]_{B M O^{\mu}(\Omega)}+a^{n}\|f\|_{L^{1}\left(B_{r}\left(x_{1}\right)\right)}
$$

hold. The same statement holds for cubes in $\Omega$ of side length $r$ and ar, respectively. 
Proof. Let $\tilde{f}:=f-f_{B_{r}\left(x_{1}\right)}$. By $\int_{B_{r}\left(x_{1}\right)} \tilde{f}-\tilde{f}_{B_{a r}\left(x_{2}\right)} d y=-\left|B_{r}\left(x_{1}\right)\right| \tilde{f}_{B_{a r}\left(x_{2}\right)}$ we obtain

$$
\left|B_{r}\left(x_{1}\right)\right|\left|\tilde{f}_{B_{a r}\left(x_{2}\right)}\right| \leq \int_{B_{r}\left(x_{1}\right)}\left|\tilde{f}-\tilde{f}_{B_{a r}\left(x_{2}\right)}\right| d y
$$

and thus

$$
\begin{aligned}
\left|B_{a r}\left(x_{2}\right)\right|[\tilde{f}]_{B M O^{\mu}} & \geq \int_{B_{a r}\left(x_{2}\right)}\left|\tilde{f}-\tilde{f}_{B_{a r}\left(x_{2}\right)}\right| d y \\
& \geq\left|B_{r}\left(x_{1}\right)\right|\left|\tilde{f}_{B_{a r}\left(x_{2}\right)}\right|
\end{aligned}
$$

which can be rewritten as

$$
\left|\tilde{f}_{B_{a r}\left(x_{2}\right)}\right| \leq a^{n}[\tilde{f}]_{B M O^{\mu}}
$$

Then we are able to estimate

$$
\begin{aligned}
& \|f\|_{L^{1}\left(B_{a r}\left(x_{2}\right)\right)} \\
\leq & \left\|f-f_{B_{r}\left(x_{1}\right)}\right\|_{L^{1}\left(B_{a r}\left(x_{2}\right)\right)}+\left|B_{a r}\left(x_{2}\right)\right|\left|f_{B_{r}\left(x_{1}\right)}\right| \\
= & \|\tilde{f}\|_{L^{1}\left(B_{a r}\left(x_{2}\right)\right)}+\left|B_{a r}\left(x_{2}\right)\right|\left|f_{B_{r}\left(x_{1}\right)}\right| \\
\leq & \left\|\tilde{f}-\tilde{f}_{B_{a r}\left(x_{2}\right)}\right\|_{L^{1}\left(B_{a r}\left(x_{2}\right)\right)}+\left|B_{a r}\left(x_{2}\right)\right|\left|\tilde{f}_{B_{a r}\left(x_{2}\right)}\right|+\frac{\left|B_{a r}\left(x_{2}\right)\right|}{\left|B_{r}\left(x_{1}\right)\right|}\|f\|_{L^{1}\left(B_{r}\left(x_{1}\right)\right)} \\
\leq & \left|B_{a r}\left(x_{2}\right)\right|[\tilde{f}]_{B M O^{\mu}}+\left|B_{a r}\left(x_{2}\right)\right| a^{n}[\tilde{f}]_{B M O^{\mu}}+a^{n}\|f\|_{L^{1}\left(B_{r}\left(x_{1}\right)\right)} \\
= & \left|B_{a r}\left(x_{2}\right)\right|\left(1+a^{n}\right)[f]_{B M O^{\mu}}+a^{n}\|f\|_{L^{1}\left(B_{r}\left(x_{1}\right)\right)} .
\end{aligned}
$$

Theorem 4. Let $\Omega \subset \mathbb{R}^{n}$ be an arbitrary domain. Let $0<\mu_{1}<\mu_{2}<\infty$. Then the seminorms $[\cdot]_{B M O^{\mu_{1}}}$ and $[\cdot]_{B M O^{\mu_{2}}}$ are equivalent.

Proof. We prove this theorem by using cubes instead of balls. Let $Q_{r}(x)$ be a cube of side length $r<\mu_{1}$ centered at $x$. We will prove that the $B M O$ seminorm in $Q_{2 r}(x)$ is controlled by the $B M O^{\mu_{1}}$ seminorm and a constant only depending on the dimension $n$ provided that $Q_{2 r}(x) \subset \Omega$. By iteration and Remark 2 we then get the stated result. Divide $Q_{2 r}(x)$ into $2^{n}$ cubes $Q_{i}$ of side length $r$ with disjoint interior such that each cube has one corner in $x$.

Assume without loss of generality that $f_{Q_{r}(x)}=0$. Then

$$
\|f\|_{L^{1}\left(Q_{r}(x)\right)} \leq \underset{8}{[f]_{B M O^{\mu}}\left|Q_{r}(x)\right|}
$$


By using Theorem 3

$$
\begin{aligned}
& \frac{1}{\left|Q_{2 r}(x)\right|} \int_{Q_{2 r}(x)}\left|f-f_{Q_{2 r}(x)}\right| d y \\
\leq & \frac{2}{\left|Q_{2 r}(x)\right|}\|f\|_{L^{1}\left(Q_{2 r}(x)\right)} \\
\leq & \frac{2}{\left|Q_{2 r}(x)\right|} \sum_{i=1}^{2^{n}}\|f\|_{L^{1}\left(Q_{i}\right)} \\
\leq & \frac{2}{\left|Q_{2 r}(x)\right|} \sum_{i=1}^{2^{n}}\left(\left|Q_{i}\right|\left(1+2^{n}\right)[f]_{B M O^{\mu_{1}}}+2^{n}\|f\|_{L^{1}\left(Q_{i} \cap Q_{r}(x)\right)}\right) \\
\leq & \frac{2}{\left|Q_{2 r}(x)\right|}\left(\left(1+2^{n}\right)[f]_{\left.B M O^{\mu_{1}}\left|Q_{2 r}(x)\right|+2^{n+1}\left|Q_{r}(x)\right|[f]_{B M O^{\mu_{1}}}\right)}\right. \\
\leq & 2\left(1+2 \cdot 2^{n}\right)[f]_{B M O^{\mu_{1}}}
\end{aligned}
$$

and thus

$$
[f]_{B M O^{2 \mu_{1}}} \leq 2\left(1+2^{n+1}\right)[f]_{B M O^{\mu_{1}}}
$$

Lemma 3. Let $\Omega \subset \mathbb{R}^{n}$ be a bounded domain, $\mu, \nu \in(1, \infty]$. Then there exists a constant $c>0$ only depending on $n, \mu, \nu$ and $\Omega$ such that for all $f \in B M O_{b}^{\mu, \nu}(\Omega)$

$$
\|f\|_{L^{1}(\Omega)} \leq c\|f\|_{B M O_{b}^{\mu, \nu}(\Omega)} .
$$

Proof. Let $\left(B_{i}\right)_{i \in I}$ be a cover of $\bar{\Omega}$ consisting of balls $B_{r}(x) \subset \Omega$ with $r<\mu$ and balls $B_{r}(x)$ with $x \in \partial \Omega$ and $r<\nu$. Then there is a finite subcover of $\bar{\Omega}$ of balls $\left(B_{i}\right)_{1 \leq i \leq N}$. This subcover contains at least one ball centered at some point on the boundary. Since there are only finitely many balls in the subcover the number

$$
r_{0}:=\min _{B_{i} \cap B_{j} \cap \Omega \neq \emptyset} \sup _{B_{r}(x) \subset B_{i} \cap B_{j} \cap \Omega} r
$$

exists and is positive. For the balls centered at the boundary we can estimate $\|f\|_{L^{1}\left(B_{i} \cap \Omega\right)} \leq\left|B_{i}\right|[f]_{b^{\nu}}$ and for all neighboring balls $B_{j}$ that are contained in $\Omega$ there is a ball $B_{r_{0}}^{i, j} \subset B_{i} \cap B_{j}$ of radius $r_{0}$ with $\|f\|_{L^{1}\left(B_{r_{0}}^{i, j}\right)} \leq\left|B_{i}\right|[f]_{b^{\nu}}$. By Theorem 3 we obtain then for the neighboring balls the estimate

$$
\|f\|_{L^{1}\left(B_{j}\right)} \leq\left|B_{j}\right|\left(1+\left(\frac{\mu}{r_{0}}\right)^{n}\right)[f]_{B M O^{\mu}}+\left(\frac{\mu}{r_{0}}\right)^{n}\left|B_{i}\right|[f]_{b^{\nu}} \leq c\|f\|_{B M O_{b}^{\mu, \nu}}
$$

and can continue this strategy until we estimated $\|f\|_{L^{1}\left(B_{j}\right)}$ on all balls $B_{j} \subset \Omega$. Thus

$$
\|f\|_{L^{1}(\Omega)} \leq c \sum_{i=1}^{N}\|f\|_{L^{1}\left(B_{i}\right)} \leq c\|f\|_{B M O_{b}^{\mu, \nu}}
$$

with some constant $c$ depending only on $n$ and the subcover $\left(B_{i}\right)_{1 \leq i \leq N}$, i.e., depending only on $n, \mu, \nu$ and $\Omega$. 
Theorem 5. Let $\Omega$ be a bounded domain and $\mu_{1}, \mu_{2}, \nu_{1}, \nu_{2} \in(0, \infty]$. Then the norms $\|\cdot\|_{B M O_{b}^{\mu_{1}, \nu_{1}}}$ and $\|\cdot\|_{B M O_{b}^{\mu_{2}, \nu_{2}}}$ are equivalent.

Proof. Assume that $\nu_{1}<\nu_{2}$. By the boundedness of $\Omega$ we have that $[f]_{B M O \infty}$ is equal to $[f]_{B M O \operatorname{diam}(\Omega)}$ such that we can assume that $\mu_{1}$ and $\mu_{2}$ are finite. By Theorem 4 we obtain the equivalence of $[f]_{B M O^{\mu_{1}}}$ and $[f]_{B M O^{\mu_{2}}}$. For $\nu_{1} \leq r<\nu_{2}$ and $x_{0} \in \partial \Omega$ we obtain by the inequality $\|f\|_{L^{1}(\Omega)} \leq c\|f\|_{B M O_{b}^{\mu_{1}, \nu_{1}}}$ of Lemma 3 the estimate

$$
\begin{aligned}
\frac{1}{r^{n}} \int_{B_{r}\left(x_{0}\right) \cap \Omega}|f(y)| d y & \leq \nu_{1}^{-n}\|f\|_{L^{1}(\Omega)} \\
& \leq c\|f\|_{B M O_{b}^{\mu_{1}, \nu_{1}}}
\end{aligned}
$$

which completes the proof.

Example 1. For the unbounded domain $\mathbb{R}_{+}=(0, \infty)$ we will give some examples that the $B M O_{b}$ norms may differ for different values of $\mu$ or $\nu$. For domains which contain arbitrarily large balls similar examples give that the spaces $B M O_{b}^{\mu, \nu}(\Omega), B M O_{b}^{\infty, \nu}(\Omega), B M O^{\mu, \infty}(\Omega)$ and $B M O_{b}^{\infty, \infty}(\Omega)$ for finite $\mu, \nu$ are different because they allow different kinds of growth at infinity.

- Let $f_{1}(x)=x$. Then $\left[f_{1}\right]_{b^{\nu}}=\frac{1}{2} \nu$ and $\left[f_{1}\right]_{B M O^{\mu}}=\frac{\mu}{4}$. This gives us that $f_{1} \in B M O_{b}^{\mu, \nu}\left(\mathbb{R}_{+}\right)$for $\mu, \nu<\infty$ but $f_{1} \notin B M O_{b}^{\mu, \nu}\left(\mathbb{R}_{+}\right)$if $\mu=\infty$ or $\nu=\infty$.

- Let $f_{2}(x)=\log (x+1)$. It is well known that $\left[f_{2}\right]_{B M O^{\infty}}<\infty$, thus $\left[f_{2}\right]_{B M O^{\mu}}<\infty$ for all $\mu \in(0, \infty]$. Furthermore, $\left[f_{2}\right]_{b^{\nu}}=$ $\frac{1}{\nu} \int_{1}^{\nu+1} \log (x) d x=\log (\nu+1)+\frac{\log (\nu+1)}{\nu}-1$. Thus $f_{2} \in B M O^{\infty, \nu}\left(\mathbb{R}_{+}\right)$ for $\nu \in(0, \infty)$ but $f_{2} \notin B M O^{\infty, \infty}\left(\mathbb{R}_{+}\right)$.

- Let

$$
f_{3}(x):= \begin{cases}x-2^{n} & : x \in\left[2^{n}, 2^{n}+\frac{1}{2^{2}} 2^{\frac{n}{2}}\right)\left(n \in \mathbb{N}_{0}\right) \\ 2^{n}+\frac{1}{2} 2^{\frac{n}{2}}-x & : x \in\left[2^{n}+\frac{1}{4} 2^{\frac{n}{2}}, 2^{n}+\frac{1}{2} 2^{\frac{n}{2}}\right)\left(n \in \mathbb{N}_{0}\right) \\ 0 & : \text { otherwise. }\end{cases}
$$

Then $\left[f_{3}\right]_{B M O^{\mu}\left(\mathbb{R}_{+}\right)} \leq \mu$ and $\left[f_{3}\right]_{b^{\infty}\left(\mathbb{R}_{+}\right)} \leq \sup _{n \in \mathbb{N}_{0}} \frac{1}{2^{n}} \int_{0}^{2^{n+1}} f_{3}(y) d y \leq$ $\frac{1}{8}$ follow from a direct calculation. Thus $f_{3} \in B M O_{b}^{\mu, \infty}\left(\mathbb{R}_{+}\right)$for $\mu<\infty$ but $f_{3} \notin B M O_{b}^{\infty, \infty}\left(\mathbb{R}_{+}\right)$which can be seen by calculating the mean oscillation in every interval $\left(2^{n}, 2^{n}+\frac{1}{2} 2^{\frac{n}{2}}\right)$.

Theorem 6. Let $\Omega \subset \mathbb{R}^{n}$ be a uniform domain with uniformly $C^{2}$-boundary

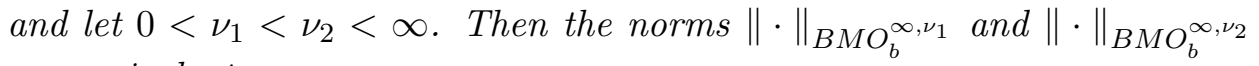
are equivalent.

Proof. We extend $f$ by Theorem 1 to $\bar{f} \in B M O^{\infty}\left(\mathbb{R}^{n}\right)$. For $\nu_{0}:=\min \left\{\frac{\nu_{1}}{8}, \frac{R^{*}}{8}\right\}$ and $\tilde{x}$ such that $B_{\nu_{0}}(\tilde{x}) \subset B_{\frac{r}{2}}\left(x_{0}\right) \cap \Omega$ for $\nu_{1} \leq r<\nu_{2}$ and $x_{0} \in \partial \Omega$ we obtain 
by Theorem 3

$$
\begin{aligned}
& \sup _{\nu_{1} \leq r<\nu_{2}, x_{0} \in \partial \Omega} \frac{1}{r^{n}} \int_{B_{r}\left(x_{0}\right) \cap \Omega}|f| d y \\
\leq & \sup _{\nu_{1} \leq r<\nu_{2}, x_{0} \in \partial \Omega} \frac{1}{r^{n}} \int_{B_{r}\left(x_{0}\right)}|\bar{f}| d y \\
\leq & \sup _{\nu_{1} \leq r<\nu_{2}, x_{0} \in \partial \Omega} \frac{c}{r^{n}}\left|B_{r}\left(x_{0}\right)\right|[\bar{f}]_{B M O^{\infty}\left(\mathbb{R}^{n}\right)}+\frac{c}{r^{n}} \int_{B_{\nu_{0}}(\tilde{x})}|f| d y \\
\leq & c[f]_{B M O^{\infty}(\Omega)}+\frac{c}{\nu_{1}^{n}} \int_{B_{\frac{\nu_{1}}{2}(\tilde{x})}|f| d y}|f| d \mid \|_{B M O^{\infty, \nu_{1}}}
\end{aligned}
$$

and for $r<\nu_{1}$ the estimate follows directly from the definition.

Theorem 7. Let $\Omega$ be a uniformly $C^{2}$-domain, $\mu \in(0, \infty], \nu_{1}, \nu_{2} \in\left(0, R^{*}\right]$ and $\nu_{1}<\nu_{2}<\infty$. Then $\|\cdot\|_{B M O_{b}^{\mu, \nu_{1}}}$ and $\|\cdot\|_{B M O_{b}^{\mu, \nu_{2}}}$ are equivalent.

Proof. By Theorem 4 and Remark 2 we can assume without loss of generality that $2 \nu_{1}=\nu_{2}<\mu$. Each $B_{r}\left(x_{0}\right) \cap \Omega$ with $x_{0} \in \partial \Omega$ and $\nu_{1} \leq r<\nu_{2}$ is a Lipschitz domain with uniform Lipschitz regularity, where $[f]_{B M O^{\mu}}\left(B_{r}\left(x_{0}\right) \cap \Omega\right)$ equals $[f]_{B M O \infty\left(B_{r}\left(x_{0}\right) \cap \Omega\right)}$. Furthermore, every $B_{r}\left(x_{0}\right) \cap \Omega$ contains a ball $B_{\nu_{1} / 4}\left(x_{1}\right)$ such that there is $r_{0}<\nu_{1}$ with $B_{\nu_{1} / 4}\left(x_{1}\right) \subset B_{r_{0}}\left(x_{0}\right)$. By Theorem 1 we obtain for every $\Omega \cap B_{r}\left(x_{0}\right)$ an extension $\bar{f}$ of $f$ such that

$$
\begin{aligned}
\|f\|_{L^{1}\left(B_{r}\left(x_{0}\right) \cap \Omega\right)} & \leq\|\bar{f}\|_{L^{1}\left(B_{r}\left(x_{0}\right)\right)} \\
& \leq c\left|B_{r}(x)\right|\left(1+8^{n}\right)[f]_{B M O^{\mu}}+8^{n}\|f\|_{L^{1}\left(B_{\nu_{1} / 4}\left(x_{1}\right)\right)}
\end{aligned}
$$

with a uniform constant $c$ since we have control on the Lipschitz regularity of $B_{r}\left(x_{0}\right) \cap \Omega$. Thus

$$
[f]_{b^{\nu_{2}}} \leq c\|f\|_{B M O_{b}^{\mu, \nu_{1}}}
$$

Theorem 8. Let $\Omega:=G \times \mathbb{R}^{n-k}$, where $G \subset \mathbb{R}^{k}$ is a bounded Lipschitz domain and $1 \leq k \leq n-1$. Let $\mu_{1}, \mu_{2}, \nu_{1}, \nu_{2} \in(0, \infty]$. Then $\|\cdot\|_{B M O_{b}^{\mu_{1}, \nu_{1}}}$ and $\|\cdot\|_{B M O_{b}^{\mu_{2}, \nu_{2}}}$ are equivalent.

Proof. Let $\delta:=\operatorname{diam}(G)$. The seminorms $[\cdot]_{B M O^{\mu_{1}}}$ and $[\cdot]_{B M O^{\mu_{2}}}$ are equivalent by $[\cdot]_{B M O^{\delta}}=[\cdot]_{B M O^{\infty}}$ for $\mu \geq \delta$ and Theorem 4 . We can assume without loss of generality that $\nu_{1}<\nu_{2}$. Let $\left\{\Omega_{i}\right\}_{i \in \mathbb{Z}^{n-k}}$ be the collection of domains

$$
G \times\left(i_{k+1} \delta,\left(i_{k+1}+1\right) \delta\right) \times \cdots \times\left(i_{n} \delta,\left(i_{n}+1\right) \delta\right)
$$

with $i \in \mathbb{Z}^{n-k}$ such that $\Omega$ is the interior of the closure of the disjoint union of all $\Omega_{i}$. Each $\Omega_{i}$ is then just the translation of the bounded Lipschitz domain $\Omega_{0}$. Since $\partial \Omega_{i} \cap \partial \Omega \neq \emptyset$ for every $i \in \mathbb{Z}^{n-k}$ we obtain by a similar argumentation as in the proof of Theorem 3 that there is a constant $C$ depending on $\nu_{1}, \mu_{1}, n$ and the shape of $\Omega_{0}$ but independent of $i$ such that

$$
\|f\|_{L^{1}\left(\Omega_{i}\right)} \leq C\|f\|_{B M O_{b}^{\mu_{1}, \nu_{1}}(\Omega)} .
$$


The number of $\Omega_{i}$ for which $\Omega_{i} \cap\left(B_{r}\left(x_{0}\right) \cap \Omega\right) \neq \emptyset$ is at most $\left(\frac{2 r+2 \delta}{\delta}\right)^{n-k}$ such that we can estimate for $\nu_{1} \leq r<\nu_{2}$ (where $\nu_{2}=\infty$ is allowed) and $x_{0} \in \partial \Omega$

$$
\begin{aligned}
\frac{1}{r^{n}} \int_{B_{r}\left(x_{0}\right) \cap \Omega}|f(y)| d y & \leq \frac{1}{r^{n}} \sum_{\Omega_{i} \cap\left(B_{r}\left(x_{0}\right) \cap \Omega\right) \neq \emptyset}\|f\|_{L^{1}\left(\Omega_{i}\right)} \\
& \leq \frac{(2 r+2 \delta)^{n-k}}{\delta^{n-k} r^{n}} C\|f\|_{B M O_{b}^{\mu_{1}, \nu_{1}}} \\
& \leq C\left(\mu_{1}, \nu_{1}, n, \delta\right)\|f\|_{B M O_{b}^{\mu_{1}, \nu_{1}}}
\end{aligned}
$$

and thus

$$
\|f\|_{B M O_{b}^{\mu_{2}, \nu_{2}}} \leq C\left(\mu_{1}, \mu_{2}, \nu_{1}, n, \delta\right)\|f\|_{B M O_{b}^{\mu_{1}, \nu_{1}}}
$$

which was left to prove.

We have shown that Jones' extension theorem does not hold for layer domains and other domains of the form $G \times \mathbb{R}^{n-k}$, where $G$ is bounded. Nevertheless, by the introduction of the $B M O_{b}$ norms, which do not allow the linear growth of $f$ as in Remark 1, we can construct a simple extension operator for $B M O_{b}$ functions.

Lemma 4. Let $\Omega:=G \times \mathbb{R}^{n-k}$ with $G \subset \mathbb{R}^{k}$ a bounded Lipschitz domain and $\mu, \nu \in(0, \infty]$. Then there is a constant $C$ depending only on $n, \Omega, \mu, \nu$ such that for each $f \in B M O_{b}^{\mu, \nu}(\Omega)$ the extension by 0 which we will denote by $\bar{f} \in B M O^{\infty}\left(\mathbb{R}^{n}\right)$ satisfies

$$
[\bar{f}]_{B M O^{\infty}\left(\mathbb{R}^{n}\right)} \leq C\|f\|_{B M O_{b}^{\mu, \nu}(\Omega)} .
$$

Proof. By Theorem 8 we can assume that $\mu=\nu=\infty$. It is immediate by construction that if $B \subset \Omega$, then $\frac{1}{|B|} \int_{B}\left|\bar{f}(y)-\bar{f}_{B}\right| d y \leq[f]_{B M O^{\infty}(\Omega)}$ and that for $B \subset \Omega^{c}, \frac{1}{|B|} \int_{B}\left|\bar{f}(y)-\bar{f}_{B}\right| d y=0$. Thus it is only left to estimate the mean oscillation in balls which have nonempty intersection with the boundary. For each $B_{r}(x)$ which satisfies $B_{r}(x) \cap \partial \Omega \neq \emptyset$ we take $x_{0} \in B_{r}(x) \cap \partial \Omega$, then $B_{r}(x) \subset B_{2 r}\left(x_{0}\right)$ and we have

$$
\frac{1}{\left|B_{r}(x)\right|} \int_{B_{r}(x)}\left|\bar{f}(y)-\bar{f}_{B_{r}(x)}\right| d y \leq \frac{2}{\left|B_{r}(x)\right|} \int_{B_{2 r}\left(x_{0}\right)}|\bar{f}(y)| d y \leq \frac{2^{n+1}}{\omega_{n}}[f]_{b^{\infty}} .
$$

Theorem 9. Miyachi's definition of the $B M O_{b}$ norm $\|\cdot\|_{B M O_{b}^{M}}$ is equivalent to $\|\cdot\|_{B M O_{b}^{\mu, \nu}}$ for $\mu, \nu \in(0, \infty]$ if $\Omega$ is a bounded Lipschitz domain.

Proof. The seminorms $[f]_{B M O^{M}}$ and $[f]_{B M O \infty}$ are equivalent by $[35$, Corollary 2.26].

For $x \in \Omega$ and $r>0$ with $B_{2 r}(x) \subset \Omega$ and $B_{5 r}(x) \cap \Omega^{c} \neq \emptyset$ let $x_{0} \in$ $\partial \Omega \cap B_{5 r}(x)$. Then $B_{r}(x) \subset B_{6 r}\left(x_{0}\right) \cap \Omega$ and

$$
\begin{aligned}
\frac{1}{\left|B_{r}(x)\right|} \int_{B_{r}(x)}|f| d y & \leq \frac{1}{\left|B_{r}(x)\right|} \int_{B_{6 r}\left(x_{0}\right) \cap \Omega}|f| d y \\
& \leq 6^{n} \omega_{n}^{-1}[f]_{b^{\infty}} .
\end{aligned}
$$


We have now proved that

$$
\|f\|_{B M O_{b}^{M}} \leq C\|f\|_{B M O_{b}^{\infty, \infty}} \leq C\|f\|_{B M O_{b}^{\mu, \nu}}
$$

where the last inequality follows from Theorem 5 .

It is now just left to estimate $[\cdot]_{b^{\nu}}$ by Miyachi's norm. First note that $f \in B M O_{b}^{\mu, \nu}(\Omega)=B M O_{b}^{\infty, \infty}(\Omega)$ can be extended by 0 to $\bar{f} \in B M O^{\infty}\left(\mathbb{R}^{n}\right)$ with $[\bar{f}]_{B M O^{\infty}\left(\mathbb{R}^{n}\right)} \leq C\|f\|_{B M O_{b}^{\mu, \nu}}$ by the same argument as in the proof of Lemma 4 . Since $\Omega$ is a bounded Lipschitz domain there exists a finite cone $K$ of height $h$ and angle $\theta$ with vertex 0 such that for every $x_{0} \in \partial \Omega$ there exists a rotation $R_{x_{0}}$ such that the cone $x_{0}+K_{x_{0}}:=x_{0}+R_{x_{0}} K$ is contained in $\Omega$. By Theorem 5 we can assume that $\nu<h$. Then there is a constant $0<c_{\theta}<$ 1 such that for all $x_{0} \in \partial \Omega$ and $0<r<\nu$ there is a ball of radius $c_{\theta} r$ with center $x \in x_{0}+K_{x_{0}}$ such that $B_{2 c_{\theta} r}(x) \subset B_{r}\left(x_{0}\right) \cap\left(x_{0}+K_{x_{0}}\right) \subset B_{r}\left(x_{0}\right) \cap \Omega$. We choose then a possibly larger ball $B_{r_{M}}(x)$ with radius $r_{M} \geq c_{\theta} r$ such that $B_{c_{\theta} r}(x) \subset B_{r_{M}}(x), B_{2 r_{M}}(x) \subset \Omega$ and $B_{5 r_{M}}(x) \cap \Omega^{c} \neq \emptyset$. Then by Theorem 3

$$
\begin{aligned}
\frac{1}{r^{n}} \int_{B_{r}\left(x_{0}\right) \cap \Omega}|f| d y \leq \frac{1}{r^{n}}\|\bar{f}\|_{L^{1}\left(B_{r}\left(x_{0}\right)\right)} \\
\leq \frac{1}{r^{n}}\left(\left|B_{r}(x)\right|\left(1+\frac{1}{c_{\theta}^{n}}\right)[\bar{f}]_{B M O^{\infty}\left(B_{r}(x)\right)}+\frac{1}{c_{\theta}^{n}}\|f\|_{L^{1}\left(B_{r_{M}}(x)\right)}\right) \\
\leq C\left([f]_{B M O^{\infty}(\Omega)}+[f]_{b^{M}(\Omega)}\right) \\
\leq C\|f\|_{B M O_{b}^{M}} .
\end{aligned}
$$

Remark 3. If we consider general domains, $f_{2}$ of Example 1 illustrates that in general $B M O_{b}^{\mu, \nu}$ may only correspond to the Miyachi norm if $\mu=\nu=\infty$ or if $B M O_{b}^{\mu, \nu}$ and $B M O_{b}^{\infty, \infty}$ are equivalent. It is easy to see that $f_{2} \notin$ $B M O_{b}^{M}\left(\mathbb{R}_{+}\right)$.

Theorem 10. Let $\Omega \subset \mathbb{R}^{n}$ be a Lipschitz half-space, i.e., a domain lying above the graph of some Lipschitz function. Then $\|\cdot\|_{B M O_{b}^{M}}$ and $\|\cdot\|_{B M O_{b}^{\infty, \infty}}$ are equivalent.

Proof. By the same arguments as in the proof of Theorem 9 one obtains that the $B M O$ seminorms are equivalent by [35, Corollary 2.26] and that $[f]_{b^{M}} \leq C[f]_{b^{\infty}}$. It is now left to prove that for all $f \in B M O_{b}^{\infty, \infty}(\Omega)$ the estimate $[f]_{b^{\infty}} \leq C\|f\|_{B M O_{b}^{M}}$ holds. This is done similarly to the argument of Theorem 9. At first we see that we can extend $f \in B M O_{b}^{\infty, \infty}$ to a $B M O^{\infty}$ function $\bar{f}$ defined on $\mathbb{R}^{n}$. Since $\Omega$ is a Lipschitz half-space, there exists an infinite cone $K$ of angle $\theta$ such that for all $x_{0} \in \partial \Omega$ the relation $x_{0}+K \subset \Omega$ holds. Then there exists a constant $c_{\theta}$ such that for all $x_{0} \in \partial \Omega$ and $r>0$ there exists a ball $B_{r_{M}}(x)$ such that $B_{2 r_{M}}(x) \subset \Omega, B_{5 r_{m}}(x) \cap \Omega^{c} \neq \emptyset$ and $B_{c_{\theta} r}(x) \subset B_{r_{M}}(x) \cap\left(B_{r}\left(x_{0}\right) \cap \Omega\right)$ with $B_{2 c_{\theta} r}(x) \subset B_{r}\left(x_{0}\right) \cap\left(x_{0}+K\right)$. Thus 
by Theorem 3

$$
\begin{aligned}
\frac{1}{r^{n}} \int_{B_{r}\left(x_{0}\right) \cap \Omega} \mid & f \mid d y \\
& \leq \frac{1}{r^{n}}\left(\left|B_{r}(x)\right|\left(1+\frac{1}{c_{\theta}^{n}}\right)[\bar{f}]_{B M O^{\infty}\left(B_{r}(x)\right)}+\frac{1}{c_{\theta}^{n}}\|f\|_{L^{1}\left(B_{r_{M}}(x)\right)}\right) \\
& \leq C\left([f]_{B M O^{\infty}(\Omega)}+[f]_{b^{M}}\right) .
\end{aligned}
$$

Remark 4. The equivalence proofs of Theorem 9 and Theorem 10 can be extended to a large class of other domains by using similar ideas. The equivalence of $B M O_{b}^{M}$ and $B M O_{b}^{\infty, \infty}$ for example also holds in exterior Lipschitz domains and domains of the form $G \times \mathbb{R}^{n-k}$, where $G \subset \mathbb{R}^{k}$ is a bounded $C^{2}$-domain, where the higher boundary regularity is needed since there is no extension operator from $B M O^{\infty}(\Omega)$ to $B M O^{\infty}\left(\mathbb{R}^{n}\right)$ (cf. Remark 1 ) such that we need to consider extension operators on subsets of $\Omega$.

Now, we want to prove an interpolation result that shows that if a function is in $B M O$ and $L^{1}$, it is also in $L^{p}$ for a large class of domains and that we can estimate it in a certain way. We will start with the result in $\mathbb{R}^{n}$.

Lemma 5. Let $f \in B M O^{\infty}\left(\mathbb{R}^{n}\right) \cap L^{1}\left(\mathbb{R}^{n}\right)$ and $1<p<\infty$. Then $f \in$ $L^{p}\left(\mathbb{R}^{n}\right)$ and the estimate

$$
\|f\|_{L^{p}\left(\mathbb{R}^{n}\right)} \leq C p\|f\|_{L^{1}\left(\mathbb{R}^{n}\right)}^{\frac{1}{p}}[f]_{B M O^{\infty}\left(\mathbb{R}^{n}\right)}^{1-\frac{1}{p}}
$$

holds, where the constant $C>0$ only depends on the dimension $n$.

Proof. Compare e.g. [19] and [27].

We will later use this lemma together with Jones' extension theorem for $B M O$-functions.

Lemma 6. Let $A \subset \mathbb{R}^{n}$ be a bounded uniform domain, $f \in L^{1}(A) \cap$ $B M O^{\infty}(A)$ and $1<p<\infty$. Let $Q_{0}$ be the largest cube in the Whitney decomposition $\mathcal{A}$ of $A$ used in the Jones' extension $\bar{f}$. Then $\bar{f}-f_{Q_{0}} \in L^{p}\left(\mathbb{R}^{n}\right)$ and

$$
\left\|\bar{f}-f_{Q_{0}}\right\|_{L^{p}\left(\mathbb{R}^{n}\right)} \leq C\left(\|\bar{f}\|_{L^{1}(B)}+\left|B \| f_{Q_{0}}\right|\right)^{\frac{1}{p}}[f]_{B M O^{\infty}(A)}^{1-\frac{1}{p}}
$$

for any point $x \in \bar{A}$, where $B=B_{(4 \sqrt{n}+2) \operatorname{diam}(A)}(x)$. If $A$ is a bounded Lipschitz domain the constant depends only on $n, p$ and the Lipschitz regularity of $A$.

Proof. We can use Theorem 1 to get $\bar{f} \in B M O^{\infty}\left(\mathbb{R}^{n}\right)$ with $[\bar{f}]_{B M O^{\infty}\left(\mathbb{R}^{n}\right)} \leq$ $C[f]_{B M O^{\infty}(A)}$. Furthermore, adding constants will not change the $B M O$ seminorm. By condition (3) on the Whitney decomposition we can see that the ball $B$ contains all cubes in $\mathcal{A}^{\prime}$ for which there exists a larger cube in $\mathcal{A}$. Thus if $y \notin B$ every cube containing $y$ corresponds to $Q_{0}$. From this we can see that $\bar{f}$ is on $B^{c}$ constantly equal to $f_{Q_{0}}$. The function $\bar{f}-f_{Q_{0}}$ has then 
compact support and is locally integrable, thus $\bar{f}-f_{Q_{0}} \in L^{1}\left(\mathbb{R}^{n}\right)$. Lemma 5 then yields

$$
\begin{aligned}
\left\|\bar{f}-f_{Q_{0}}\right\|_{L^{p}\left(\mathbb{R}^{n}\right)} & \leq C\left\|\bar{f}-f_{Q_{0}}\right\|_{L^{1}\left(\mathbb{R}^{n}\right)}^{\frac{1}{p}}\left[\bar{f}-f_{Q_{0}}\right]_{B M O^{\infty}\left(\mathbb{R}^{n}\right)}^{1-\frac{1}{p}} \\
& \leq C\left\|\bar{f}-f_{Q_{0}}\right\|_{L^{1}(B)}^{\frac{1}{p}}[\bar{f}]_{B M O^{\infty}\left(\mathbb{R}^{n}\right)}^{1-\frac{1}{p}} \\
& \leq C\left(\|\bar{f}\|_{L^{1}(B)}+|B|\left|f_{Q_{0}}\right|\right)^{\frac{1}{p}}[f]_{B M O^{\infty}(A)}^{1-\frac{1}{p}}
\end{aligned}
$$

Theorem 11. Let $A$ be a bounded uniform domain, $f \in L^{1}(A) \cap B M O^{\infty}(A)$ and $1<p<\infty$. Then $f \in L^{p}(A)$ with

$$
\|f\|_{L^{p}(A)} \leq C\left(\left(1+\frac{\operatorname{diam}(A)^{n}}{\left|Q_{0}\right|}\right)\|f\|_{L^{1}(A)}\right)^{\frac{1}{p}}[f]_{B M O \infty(A)}^{1-\frac{1}{p}}+\frac{|A|^{\frac{1}{p}}}{\left|Q_{0}\right|}\|f\|_{L^{1}(A)} .
$$

If $A$ is a bounded Lipschitz domain the constant $C>0$ depends only on $n, p$ and the Lipschitz regularity of $A$.

Proof. Note that by Lemma 2 all $Q_{j}^{\prime} \in \mathcal{A}^{\prime}$ that correspond to $Q_{j} \neq Q_{0}$ are contained in a cube of side length $\left(130 K^{2}+2\right) \ell\left(Q_{j}\right)$ with the same center as $Q_{j}$. Thus for $B:=B_{(4 \sqrt{n}+2) \operatorname{diam}(A)}(x)$ we have

$$
\|\bar{f}\|_{L^{1}(B)} \leq\left(\left(130 K^{2}+2\right)^{n}+1\right)\|f\|_{L^{1}(A)}
$$

because there are at most $\left(130 K^{2}+2\right)^{n}$ cubes outside of $Q_{j}$, in which $f$ may be defined as $f_{Q_{j}}$. By the previous lemma we get

$$
\begin{aligned}
& \|f\|_{L^{p}(A)} \\
& \quad \leq\left\|\bar{f}-f_{Q_{0}}\right\|_{L^{p}\left(\mathbb{R}^{n}\right)}+|A|^{\frac{1}{p}}\left|f_{Q_{0}}\right| \\
& \leq C\left(\|\bar{f}\|_{L^{1}(B)}+\left|B \| f_{Q_{0}}\right|\right)^{\frac{1}{p}}[f]_{B M O^{\infty}(A)}^{1-\frac{1}{p}}+|A|^{\frac{1}{p}}\left|f_{Q_{0}}\right| \\
& \leq C\left(\left(\left(130 K^{2}+2\right)^{n}+1\right)\|f\|_{L^{1}(A)}+\frac{|B|}{\left|Q_{0}\right|}\|f\|_{L^{1}(A)}\right)^{\frac{1}{p}}[f]_{B M O^{\infty}(A)}^{1-\frac{1}{p}} \\
& \quad+\frac{|A|^{\frac{1}{p}}}{\left|Q_{0}\right|}\|f\|_{L^{1}(A)} \\
& \leq C(K, n, p)\left(\left(1+\frac{\operatorname{diam}(A)^{n}}{\left|Q_{0}\right|}\right)\|f\|_{L^{1}(A)}\right)^{\frac{1}{p}}[f]_{B M O^{\infty}(A)}^{1-\frac{1}{p}}+\frac{|A|^{\frac{1}{p}}}{\left|Q_{0}\right|}\|f\|_{L^{1}(A)} .
\end{aligned}
$$

Theorem 12. Let $\Omega \subset \mathbb{R}^{n}$ be a bounded uniform domain. Let $\mu, \nu \in(0, \infty]$ and $p \in[1, \infty)$. Then the embeddings

$$
\begin{gathered}
\operatorname{BMO}_{b}^{\mu, \nu}(\Omega) \hookrightarrow L^{p}(\Omega), \\
V M O_{b, 0, \sigma}^{\mu, \nu}(\Omega) \hookrightarrow L_{\sigma}^{p}(\Omega)
\end{gathered}
$$

hold. 
Proof. From Lemma 3 we see that $\|f\|_{L^{1}(\Omega)} \leq C\|f\|_{B M O_{b}^{\mu, \nu}}$ and by definition $[f]_{B M O^{\mu}(\Omega)} \leq\|f\|_{B M O_{b}^{\mu, \nu}}$. By the equivalence result for different finite $\mu$ of Theorem 4 we get that we can replace $[f]_{B M O^{\mu}(\Omega)}$ by $[f]_{B M O^{\text {diam }}(\Omega)(\Omega)}=$ $[f]_{B M O \infty(\Omega)}$. Then we can use Theorem 11 in order to get

$$
\begin{aligned}
\|f\|_{p} & \leq C\left(\|f\|_{L^{1}(\Omega)}^{\frac{1}{p}}[f]_{B M O^{\infty}}^{1-\frac{1}{p}}+\|f\|_{L^{1}(\Omega)}\right) \\
& \leq C\|f\|_{B M O_{b}^{\mu, \nu}}
\end{aligned}
$$

Finally we will give an equivalence result of $B M O_{b}^{\mu, \nu} p$ for different $p$. Our proof here will be based on Jones' extension theorem for $B M O$-functions. Another proof for this fact can be found in [3].

Theorem 13. Let $\mu \in(0, \infty]$ and $\Omega \subset \mathbb{R}^{n}$ be a uniformly $C^{2}$-domain. Let $\nu \in\left(0, R^{*}\right)$ and $p \in(1, \infty)$. If $\mu<\infty$, then we assume additionally $\nu<\infty$. Then $\|\cdot\|_{B M O_{b}^{\mu, \nu} p}$ and $\|\cdot\|_{B M O_{b}^{\mu, \nu}}$ are equivalent.

Proof. The seminorms $[\cdot]_{B M O^{\mu} p}$ and $[\cdot]_{B M O^{\mu}}$ are equivalent by the JohnNirenberg inequality ([22]) and Hölder's inequality. By Theorem 4 we can furthermore assume that $\mu>\nu$ if $\nu$ is finite. By Hölder's inequality

$$
\begin{aligned}
{[f]_{b^{\nu}} } & =\sup _{x_{0} \in \partial \Omega, r<\nu} \frac{1}{r^{n}} \int_{B_{r}\left(x_{0}\right) \cap \Omega}|f| d y \\
& \leq \sup _{x_{0} \in \partial \Omega, r<\nu} \omega_{n}^{\frac{1}{p^{\prime}}}\left(\frac{1}{r^{n}} \int_{B_{r}\left(x_{0}\right) \cap \Omega}|f|^{p} d y\right)^{1 / p} \\
& =\omega_{n}^{\frac{1}{p^{\prime}}}[f]_{b^{\nu} p} .
\end{aligned}
$$

Thus it is left to show that there is a constant $C>0$ such that for all $x_{0} \in \partial \Omega, r<\nu$ and $f \in B M O_{b}^{\mu, \nu}(\Omega)$ the estimate

$$
\frac{1}{r^{n}} \int_{B_{r}\left(x_{0}\right) \cap \Omega}|f|^{p} \leq C\|f\|_{B M O_{b}^{\mu, \nu}}^{p}
$$

holds. By the assumption $r<\nu<R^{*}$ we see that all domains $B_{r}\left(x_{0}\right) \cap \Omega$ with $r<\nu$ and $x_{0} \in \partial \Omega$ are Lipschitz domains, where we can estimate the Lipschitz regularity uniformly in $r$ and $x_{0}$. Since we assumed $\mu>\nu$ the seminorms $[\cdot]_{B M O^{\mu}\left(B_{r}\left(x_{0}\right) \cap \Omega\right)}$ and $[\cdot]_{B M O^{\infty}\left(B_{r}\left(x_{0}\right) \cap \Omega\right)}$ coincide. Then for every $f \in B M O_{b}^{\mu, \nu}(\Omega)$ the restriction $\left.f\right|_{B_{r}\left(x_{0}\right) \cap \Omega}$ is in $\left.B M O^{\infty}\left(B_{r}\left(x_{0}\right) \cap \Omega\right)\right) \cap$ $\left.L^{1}\left(B_{r}\left(x_{0}\right) \cap \Omega\right)\right)$ such that we can apply Theorem 11 to obtain

$$
\begin{aligned}
& \|f\|_{L^{p}\left(B_{r}\left(x_{0}\right) \cap \Omega\right)} \\
& \qquad C\left(\left(\left(1+\frac{r^{n}}{\left|Q_{0}\right|}\right)\|f\|_{\left.L^{1}\left(B_{r}\left(x_{0}\right) \cap \Omega\right)\right)}\right)^{\frac{1}{p}}\right. \\
& {[f]_{B M O^{\infty}\left(B_{r}\left(x_{0}\right) \cap \Omega\right)}^{1-\frac{1}{p}}} \\
& \left.+\frac{r^{\frac{n}{p}}}{\left|Q_{0}\right|}\|f\|_{L^{1}\left(B_{r}\left(x_{0}\right) \cap \Omega\right)}\right) .
\end{aligned}
$$


By the assumption on the Whitney decomposition and $r<R^{*}$ we obtain that $Q_{0}$ is at least of side length $\frac{r}{16 \sqrt{n}}$ and thus we can rewrite the above inequality by

$$
\begin{aligned}
& \|f\|_{\left.L^{p}\left(B_{r}\left(x_{0}\right) \cap \Omega\right)\right)} \\
\leq & C\|f\|_{L^{1}\left(B_{r}\left(x_{0}\right) \cap \Omega\right)}^{\frac{1}{p}}[f]_{B M O^{\infty}\left(B_{r}\left(x_{0}\right) \cap \Omega\right)}^{1-\frac{1}{p}}+C r^{n\left(\frac{1}{p}-1\right)}\|f\|_{L^{1}\left(B_{r}\left(x_{0}\right) \cap \Omega\right)} \\
\leq & C r^{\frac{n}{p}}[f]_{b^{\nu}}^{\frac{1}{p}}[f]_{B M O^{\mu}(\Omega)}^{1-\frac{1}{p}}+C r^{\frac{n}{p}}[f]_{b^{\nu}}
\end{aligned}
$$

from which we can conclude that

$$
\begin{aligned}
{[f]_{b^{\nu} p} } & =\sup _{x_{0} \in \partial \Omega, r<\nu} r^{-\frac{n}{p}}\|f\|_{L^{p}\left(B_{r}\left(x_{0}\right) \cap \Omega\right)} \\
& \leq C\|f\|_{B M O_{b}^{\mu, \nu}}
\end{aligned}
$$

Remark 5. The function $f_{3}$ of Example 1 shows that it is in fact necessary to exclude the case $\mu<\infty$ and $\nu=\infty$ in the case of the half space since $\left[f_{3}\right]_{b^{\infty} p\left(\mathbb{R}_{+}\right)}=\infty$ for $p \in(1, \infty)$.

Theorem 14. Let $\Omega \subset \mathbb{R}^{n}$ be an arbitrary domain and let $p \in(1, \infty)$. Then the norms $\|\cdot\|_{B M O_{b}^{M}}$ and $\|\cdot\|_{B M O_{b}^{M} p}$ are equivalent.

Proof. The proof of this theorem uses the same ideas as the proof of Theorem 13. By the John-Nirenberg inequality $[\cdot]_{B M O^{M}}$ and $[\cdot]_{B M O^{M}}$ are equivalent and it follows from Hölder's inequality that $[f]_{b^{M}} \leq C[f]_{b^{M}}$. We have now a look at all balls $B:=B_{r}(x)$ such that $B_{2 r}(x) \subset \Omega$ and $B_{5 r}(x) \cap \Omega^{c} \neq \emptyset$. Since the constant of Theorem 11 and the ratio $\frac{|B|}{\left|Q_{0}\right|}$ are scale invariant and we are only considering balls here we have

$$
\begin{aligned}
\frac{1}{|B|^{\frac{1}{p}}}\|f\|_{L^{p}(B)} & \leq C\left(\left(1+\frac{r^{n}}{\left|Q_{0}\right|}\right) \frac{1}{|B|}\|f\|_{L^{1}(B)}\right)^{\frac{1}{p}}[f]_{B M O^{\infty}(B)}^{1-\frac{1}{p}}+\frac{1}{\left|Q_{0}\right|}\|f\|_{L^{1}(B)} \\
& \leq C[f]_{b^{M}}^{\frac{1}{p}}[f]_{B M O^{M}}^{1-\frac{1}{p}}+C[f]_{b^{M}} .
\end{aligned}
$$

Thus we have proved that $[f]_{b^{M} p} \leq C\|f\|_{B M O_{b}^{M}}$.

Remark 6. Our definition of $b^{\nu} p$ is slightly different from those in [2], [3], [4]. In these papers the restriction on $B_{r}(x)$ centered at $x$ on the boundary is

$$
B_{r}(x) \subset U_{\nu}(\partial \Omega)=\left\{x \in \mathbf{R}^{n} \mid \operatorname{dist}(x, \partial \Omega)<\nu\right\}
$$

instead of $r<\nu$. If $\nu$ is smaller than or equal to the reach $R^{*}$, then this condition is equivalent to $r<\nu$. Otherwise, $B_{r}(x) \subset U_{\nu}(\partial \Omega)$ is actually weaker. For example, consider $\Omega=\operatorname{int} B_{2}(0) \backslash B_{1}(0)$ and $\nu=1.1$ to get $U_{\nu}(\partial \Omega)=\operatorname{int} B_{3.1}(0)$. The ball $B_{2}(x)$ for $x \in \partial B_{1}(0)$ is still contained in $U_{\nu}(\partial \Omega)$ although $2>\nu$. The definition in the present paper is convenient to handle the case $\nu>R^{*}$. 


\section{The heat Semigroup in $B M O$-Type SPACES}

In this section we will prove several properties of the heat semigroup with respect to the considered $B M O_{b}$ spaces, i.e., we consider the equation

$$
\begin{array}{rll}
u_{t}-\Delta u & =0 & \text { in } \Omega \times(0, T), \\
u & =0 & \text { in } \partial \Omega \times(0, T), \\
u(0) & =u_{0} . &
\end{array}
$$

We will start with the case $\Omega=\mathbb{R}^{n}$ and $T=\infty$.

Theorem 15. Let $u_{0} \in B M O^{\infty}\left(\mathbb{R}^{n}\right)$. Then there is a solution $u$ to (4.1) which satisfies the estimate

$$
\sup _{t>0}\left([u(t)]_{B M O^{\infty}}+t^{\frac{1}{2}}\|\nabla u(t)\|_{\infty}+t\left\|\nabla^{2} u(t)\right\|_{\infty}+t\left\|u_{t}(t)\right\|_{\infty}\right) \leq C\left[u_{0}\right]_{B M O^{\infty}}
$$

with a constant $C>0$ just depending on $n$.

Proof. We will derive the estimate $\sup _{t>0}[u(t)]_{B M O^{\infty}} \leq C\left[u_{0}\right]_{B M O^{\infty}}$ by duality. Let $\varphi \in \mathcal{H}^{1}\left(\mathbb{R}^{n}\right)$, where $\mathcal{H}^{1}\left(\mathbb{R}^{n}\right)$ is the Hardy space which is defined as

$$
\left\{f \in L^{1}\left(\mathbb{R}^{n}\right):\|f\|_{\mathcal{H}^{1}}:=\left\|\sup _{s>0}\left|G_{s} * f\right|\right\|_{1}<\infty\right\}
$$

We define $(u(t), \varphi)=\left(u_{0}, G_{t} * \varphi\right)$ as a pairing of $B M O^{\infty}$ and $\mathcal{H}^{1}$ to get

$$
\begin{aligned}
|(u(t), \varphi)| & \leq\left[u_{0}\right]_{B M O^{\infty}}\left\|G_{t} * \varphi\right\|_{\mathcal{H}^{1}} \\
& =\left[u_{0}\right]_{B M O^{\infty}} \sup _{s>0}\left\|G_{s} * G_{t} * \varphi\right\|_{L^{1}} \\
& \leq\left[u_{0}\right]_{B M O^{\infty}}\|\varphi\|_{\mathcal{H}^{1}} .
\end{aligned}
$$

The desired estimate follows from the duality $\left(\mathcal{H}^{1}\right)^{*}=B M O^{\infty}$. The inequality can also be derived from the estimate

$$
[f * g]_{B M O \infty} \leq\|g\|_{1}[f]_{B M O \infty} \quad\left(f \in B M O^{\infty}\left(\mathbb{R}^{n}\right), g \in L^{1}\left(\mathbb{R}^{n}\right)\right),
$$

which was proved in [18] (equation (41)) by a similar duality argument.

The derivative estimates are also proved via a duality argument. The gradient estimate $\|\nabla u(t)\|_{\infty} \leq t^{1 / 2}\left[u_{0}\right]_{B M O} \infty$ has already been proved in the appendix of [2]. We will here just prove the estimate for the second derivative $\nabla^{2} u$, which is done by using the same ideas as the proof in the appendix of [2]. The estimate for the first derivative can be proved in a similar way. The time derivative estimate follows then from the estimate on the second derivative by $u_{t}=\Delta u$. As a first step we prove the estimate

$$
t\left\|\nabla^{2} G_{t} * u_{0}\right\|_{\mathcal{H}^{1}} \leq C\left\|u_{0}\right\|_{1}
$$


for all $u_{0} \in L^{1}\left(\mathbb{R}^{n}\right)$ for the special case $t=1$. By the definition of the $\mathcal{H}^{1}$-norm

$$
\begin{aligned}
\left\|\partial_{i} \partial_{j} G_{1} * u_{0}\right\|_{\mathcal{H}^{1}} & =\left\|\sup _{s>0}\left|G_{s} * \partial_{i} \partial_{j} G_{1} * u_{0}\right|\right\|_{1} \\
& \leq\left\|\sup _{s>0}\left(\left|\partial_{i} \partial_{j} G_{s+1}\right| *\left|u_{0}\right|\right)\right\|_{1} \\
& \left.\leq \| \sup _{s>0}\left|\partial_{i} \partial_{j} G_{s+1}\right|\right) *\left|u_{0}\right| \|_{1} \\
& \leq\left\|\sup _{s>0}\left|\partial_{i} \partial_{j} G_{s+1}\right|\right\|_{1}\left\|u_{0}\right\|_{1} .
\end{aligned}
$$

Since $\partial_{i} \partial_{j} G_{t}=-\delta_{i j} \frac{G_{t}}{2 t}+x_{i} x_{j} \frac{G_{t}}{4 t^{2}}$, we obtain for $\varrho=\frac{|x|^{2}}{4 t}$ the estimate

$$
\left|\partial_{i} \partial_{j} G_{t}(x)\right| \leq \frac{2 \delta_{i j}}{\pi^{\frac{n}{2}}} \frac{1}{|x|^{n+2}} \mathrm{e}^{-\varrho} \varrho^{\frac{n}{2}+1}+\frac{4}{\pi^{\frac{n}{2}}} \frac{\left|x_{i}\right|\left|x_{j}\right|}{|x|^{n+4}} \mathrm{e}^{-\varrho} \varrho^{\frac{n}{2}+2}
$$

from which we can conclude that

$$
\left|\partial_{i} \partial_{j} G_{t}(x)\right| \leq \frac{C_{0}}{|x|^{n+2}} .
$$

Furthermore, for $t \geq 1$ we can estimate $\left|\partial_{i} \partial_{j} G_{t}(x)\right| \leq 2(4 \pi)^{-\frac{n}{2}}$ such that we have

$$
\left|\partial_{i} \partial_{j} G_{s+1}(x)\right| \leq \min \left\{2(4 \pi)^{-\frac{n}{2}}, \frac{C_{0}}{|x|^{n+2}}\right\}=: a(x) \text { for } s>0, x \in \mathbb{R}^{n} .
$$

Since $a \in L^{1}\left(\mathbb{R}^{n}\right)$, we get with $C_{*}=\int_{\mathbb{R}^{n}} a(x) d x$ the estimate

$$
\left\|\partial_{i} \partial_{j} G_{1} * u_{0}\right\|_{\mathcal{H}^{1}} \leq C_{*}\left\|u_{0}\right\|_{1}
$$

which is (4.3) for $t=1$. In order to generalize this to arbitrary time $t>0$ we rescale $u$ by the scaling transformation $u_{\lambda}(x)=\lambda^{n} u(\lambda x)$ for $\lambda>0$. The norms in $L^{1}\left(\mathbb{R}^{n}\right)$ and $\mathcal{H}^{1}\left(\mathbb{R}^{n}\right)$ are invariant under this transformation and thus we get from the equality $\left(\partial_{i} \partial_{j} G_{1}\right) *\left(u_{0}\right)_{\lambda}=\lambda^{2}\left(\left(\partial_{i} \partial_{j} G_{\lambda^{2}}\right) * u_{0}\right)_{\lambda}$ and the estimate for $t=1$ that

$$
\lambda^{2}\left\|\left(\partial_{i} \partial_{j} G_{\lambda^{2}} * u_{0}\right)\right\|_{\mathcal{H}^{1}} \leq C_{*}\left\|u_{0}\right\|_{1} .
$$

We obtain now (4.3) for $t>0$ by taking $\lambda=t^{\frac{1}{2}}$. Then by duality

$$
\left\|\partial_{i} \partial_{j} G_{t} * u_{0}\right\|_{\infty} \leq C t^{-1}\left[u_{0}\right]_{B M O^{\infty}}
$$

for all $t>0$.

Similar estimates can be obtained for the half space via an odd extension and reduction to the case $\Omega=\mathbb{R}^{n}$. We will first formulate the extension argument.

Lemma 7. Let $\mu>0$ and $\nu \geq 2 \mu$. Then there exists a dimensional constant $C>0$ such that for all $f \in B M O_{b}^{\mu, \nu}\left(\mathbb{R}_{+}^{n}\right)$ the odd extension $\bar{f} \in B M O^{\mu}\left(\mathbb{R}^{n}\right)$ satisfies

$$
[\bar{f}]_{B M O^{\mu}\left(\mathbb{R}^{n}\right)} \leq C\|f\|_{B M O_{b}^{\mu, \nu}\left(\mathbb{R}_{+}^{n}\right)},
$$

where the odd extension $\bar{f}$ is defined by $\bar{f}(x)=f(x)$ if $x_{n}>0, \bar{f}(x)=0$ if $x_{n}=0$ and $\bar{f}(x)=-f\left(x_{1}, \ldots, x_{n-1},-x_{n}\right)$ if $x_{n}<0$. 
Proof. Let $x \in \mathbb{R}^{n}$ and $r<\mu$. We distinguish between two cases. If $B_{r}(x) \subset$ $\mathbb{R}_{+}^{n}$ or $B_{r}(x) \subset\left(\mathbb{R}_{+}^{n}\right)^{c}$, then $\frac{1}{\left|B_{r}(x)\right|} \int_{B_{r}(x)}\left|\bar{f}(y)-\bar{f}_{B_{r}(x)}\right| d y \leq[f]_{B M O^{\mu}\left(\mathbb{R}_{+}^{n}\right)}$. If $B_{r}(x) \cap \partial \mathbb{R}_{+}^{n} \neq \emptyset$, then there is $\tilde{x} \in B_{r}(x)$ with $\tilde{x}_{n}=0$. Since $\tilde{x} \in B_{r}(x)$ the relation $B_{r}(x) \subset B_{2 r}(\tilde{x})$ holds and thus by $2 r<\nu$

$$
\begin{aligned}
\frac{1}{\left|B_{r}(x)\right|} \int_{B_{r}(x)}\left|\bar{f}(y)-\bar{f}_{B_{r}(x)}\right| d y & \leq \frac{2}{\omega_{n} r^{n}} \int_{B_{2 r}(\tilde{x})}|\bar{f}(y)| d y \\
& \leq \frac{2^{n+1}}{\omega_{n}}[f]_{b^{\nu}} .
\end{aligned}
$$

The conclusion of the lemma holds in particular for the odd extension from $B M O_{b}^{\infty, \infty}\left(\mathbb{R}_{+}^{n}\right)$ to $B M O^{\infty}\left(\mathbb{R}^{n}\right)$. For the case $\mu=\infty, \nu<\infty$ this extension does not hold. The function $f(x):=\log |x+(0, \ldots, 0,1)|$ is in $B M O_{b}^{\infty, \nu}\left(\mathbb{R}_{+}^{n}\right)$ for finite $\nu$ but the odd extension is not in $B M O^{\infty}\left(\mathbb{R}^{n}\right)$ since for $x$ with $x_{n}=0$

$$
\frac{1}{\left|B_{r}(x)\right|} \int_{B_{r}(x)}\left|\bar{f}(y)-\bar{f}_{B_{r}(x)}\right| d y=\frac{1}{\left|B_{r}(x)\right|} \int_{B_{r}(x)}|\bar{f}(y)| d y \rightarrow \infty \quad(r \rightarrow \infty) .
$$

Theorem 16. Let $u_{0} \in B M O_{b}^{\infty, \infty}\left(\mathbb{R}_{+}^{n}\right)$. Then there is a solution $u$ to (4.1) which satisfies the estimate

$$
\begin{array}{r}
\sup _{t>0}\left(\|u(t)\|_{B M O_{b}^{\infty, \infty}}+t^{1 / 2}\|\nabla u(t)\|_{\infty}+t\left\|\nabla^{2} u(t)\right\|_{\infty}+t\left\|u_{t}(t)\right\|_{\infty}\right) \\
\leq C\left\|u_{0}\right\|_{B M O_{b}^{\infty, \infty}}
\end{array}
$$

with a constant $C$ just depending on $n$. In particular, the corresponding operator $H: u_{0} \mapsto H(t) u_{0}=u(\cdot, t)$ is a bounded analytic semigroup in $B M O_{b}^{\infty, \infty}\left(\mathbb{R}_{+}^{n}\right)$.

Proof. By Lemma 7 we can extend $u_{0}$ to $\bar{u}_{0} \in B M O^{\infty}\left(\mathbb{R}^{n}\right)$, which is a function that is odd with respect to the last component. We can now use Theorem 15 to get a solution $\bar{u}$ to (4.1) with $\Omega=\mathbb{R}^{n}$ and initial data $\bar{u}_{0}$. The solution $\bar{u}$ then is also an odd function in the last component and satisfies the estimate

$$
\begin{array}{r}
\sup _{t>0}\left([\bar{u}(t)]_{B M O^{\infty}\left(\mathbb{R}^{n}\right)}+t^{\frac{1}{2}}\|\nabla \bar{u}(t)\|_{L^{\infty}\left(\mathbb{R}^{n}\right)}+t\left\|\nabla^{2} \bar{u}(t)\right\|_{L^{\infty}\left(\mathbb{R}^{n}\right)}+t\left\|\bar{u}_{t}(t)\right\|_{L^{\infty}\left(\mathbb{R}^{n}\right)}\right) \\
\leq C\left[\bar{u}_{0}\right]_{B M O \infty\left(\mathbb{R}^{n}\right)} \leq C\left\|u_{0}\right\|_{B M O_{b}^{\infty, \infty}\left(\mathbb{R}_{+}^{n}\right)} .
\end{array}
$$

Then $u(t):=\left.\bar{u}(t)\right|_{\mathbb{R}_{+}^{n}}$ satisfies (4.1) with initial data $u_{0}$. The boundary condition is satisfied because $\bar{u}$ is an odd function in the last component.

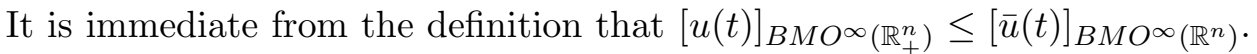
Furthermore, we obtain for $r>0$ and $x_{0} \in \partial \mathbb{R}_{+}^{n}$

$$
\begin{aligned}
\frac{1}{r^{n}} \int_{B_{r}\left(x_{0}\right) \cap \mathbb{R}_{+}^{n}}|u(y, t)| d y & =\frac{1}{2 r^{n}} \int_{B_{r}\left(x_{0}\right)}|\bar{u}(y, t)| d y \\
& =\frac{1}{2 r^{n}} \int_{B_{r}\left(x_{0}\right)}\left|\bar{u}(y, t)-\bar{u}_{B_{r}\left(x_{0}\right)}(t)\right| d y \\
& \leq \frac{\omega_{n}}{2}[\bar{u}(t)]_{B M O^{\infty}\left(\mathbb{R}^{n}\right)}
\end{aligned}
$$


and thus $[u(t)]_{b^{\infty}\left(\mathbb{R}_{+}^{n}\right)} \leq C\left\|u_{0}\right\|_{B M O_{b}^{\infty, \infty}\left(\mathbb{R}_{+}^{n}\right)}$.

If the underlying geometry of the domain is more complicated or one of the parameters is finite, we need a different method to prove similar estimates.

Lemma 8. Let $n<p<\infty$. If $u_{0} \in C_{c}^{\infty}(\Omega)$ and $\Omega$ is a uniformly $C^{3}$ domain, then there is a solution $u$ of $(4.1)$ with $u(t) \in C^{2}(\bar{\Omega}) \cap W_{0}^{1, p}(\Omega)$ for all $t>0$ satisfying

$$
\sup _{0<t<T_{0}}\left(\|u(t)\|_{\infty}+t^{\frac{1}{2}}\|\nabla u(t)\|_{\infty}+t\left\|\nabla^{2} u(t)\right\|_{\infty}+t\left\|u_{t}(t)\right\|_{\infty}\right)<\infty
$$

for every $0<T_{0}<\infty$.

Proof. By Theorem 3.1.2 in [30] there exists an analytic semigroup $H$ in $L^{p}(\Omega)$ to $(4.1)$. We define $u(t):=H(t) u_{0}$. We argue in a similar way as in the proof of Proposition 5.2 in [1], where a similar property was shown for the Stokes semigroup. By the semigroup properties we obtain an estimate

$$
\sup _{0<t<T_{0}}\left(\|u(t)\|_{D\left(\Delta_{p}\right)}+t\left\|u_{t}(t)\right\|_{D\left(\Delta_{p}\right)}\right) \leq C_{T_{0}}\left\|u_{0}\right\|_{D\left(\Delta_{p}\right)},
$$

where $\|f\|_{D\left(\Delta_{p}\right)}=\|f\|_{p}+\|\Delta f\|_{p}$. This norm is equivalent to $\|f\|_{W^{2, p}}$ and thus we have by $u_{0} \in C_{c}^{\infty}(\Omega) \subset D\left(\Delta_{p}\right)$

$$
\sup _{0<t<T_{0}}\|u(t)\|_{1, p}+t^{\frac{1}{2}}\|\nabla u(t)\|_{1, p}+t\left\|u_{t}(t)\right\|_{1, p}<\infty .
$$

For estimating $\left\|\nabla^{2} u\right\|_{W^{1, p}}$ we note that $u$ solves the equation $\Delta u=u_{t}$ in $\Omega$ with $u=0$ on $\partial \Omega$. Since $\Omega$ is a $C^{3}$-domain we obtain by higher regularity theory for elliptic systems as in Theorem 8.13 of [17] for $t \leq T_{0}$

In summary we have that

$$
\begin{aligned}
\|u(t)\|_{3, p} & \leq C\left(\left\|u_{t}(t)\right\|_{1, p}+\|u(t)\|_{p}\right) \\
& \leq \frac{1}{t} C_{T_{0}}\left\|u_{0}\right\|_{D\left(\Delta_{p}\right)} .
\end{aligned}
$$

$$
\begin{aligned}
& \sup _{0<t<T_{0}}\left(\|u(t)\|_{\infty}+t^{\frac{1}{2}}\|\nabla u(t)\|_{\infty}+t\left\|\nabla^{2} u(t)\right\|_{\infty}+t\left\|u_{t}(t)\right\|_{\infty}\right) \\
\leq & \sup _{0<t<T_{0}}\left(\|u(t)\|_{1, p}+t^{\frac{1}{2}}\|\nabla u(t)\|_{1, p}+t\left\|\nabla^{2} u(t)\right\|_{1, p}+t\left\|u_{t}(t)\right\|_{1, p}\right) \\
<\infty, &
\end{aligned}
$$

$u(t) \in C^{2}(\bar{\Omega})$ by the Sobolev embedding theorem and $u(t) \in W_{0}^{1, p}(\Omega)$ by the boundary conditions on $u$.

Theorem 17. Let $\Omega$ be a domain with uniformly $C^{3}$-boundary. Let $\mu, \nu \in$ $(0, \infty]$. Then there exist constants $C>0$ and $T_{0}>0$ such that for all $u_{0} \in V M O_{b, 0}^{\mu, \nu}(\Omega)$ there is a solution $u$ to (4.1) satisfying

$$
\sup _{0<t<T_{0}}\left(t^{\frac{1}{2}}\|\nabla u(t)\|_{\infty}+t\left\|\nabla^{2} u(t)\right\|_{\infty}+t\left\|u_{t}(t)\right\|_{\infty}\right) \leq C\left\|u_{0}\right\|_{B M O_{b}^{\mu, \nu}}
$$

Proof. The proof is similar to the proof of the same estimate for the Stokes equations (cf. [1], [2]). By Lemma 8 there are solutions satisfying (4.5) for every $u_{0} \in C_{c}^{\infty}(\Omega)$. Let

$$
N(u)(x, t):=t^{\frac{1}{2}}|\nabla u(x, t)|+t\left|\nabla^{2} u(x, t)\right|+t\left|u_{t}(x, t)\right| .
$$


We assume for these solutions that the estimate does not hold. Then there is a sequence of solutions $u^{m}$ to initial data $u_{0}^{m} \in C_{c}^{\infty}(\Omega)$ and a sequence $t_{m} \rightarrow 0$ such that

$$
\left\|N\left(u^{m}\right)\left(\cdot, t_{m}\right)\right\|_{\infty}>m\left\|u_{0}^{m}\right\|_{B M O_{b}^{\mu, \nu}} .
$$

We normalize $u^{m}$ by $\tilde{u}^{m}:=u^{m} /\left(\sup _{0<t<t_{m}}\left\|N\left(u^{m}\right)(\cdot, t)\right\|_{\infty}\right)$ and thus obtain

$$
\sup _{0<t<t_{m}}\left\|N\left(\tilde{u}^{m}\right)(\cdot, t)\right\|_{\infty}=1
$$

and

$$
\left\|\tilde{u}_{0}^{m}\right\|_{B M O_{b}^{\mu, \nu}}<1 / m .
$$

Thus there exist $x_{m} \in \Omega$ and $\tau_{m}<t_{m}$ such that $N\left(\tilde{u}^{m}\right)\left(x_{m}, \tau_{m}\right) \geq 1 / 2$. Then we rescale the solution with respect to $\left(x_{m}, \tau_{m}\right)$ by

$$
v^{m}(x, t):=\tilde{u}^{m}\left(\tau_{m}^{1 / 2} x+x_{m}, \tau_{m} t\right) \quad v_{0}^{m}(x)=\tilde{u}_{0}^{m}\left(t_{m}^{1 / 2} x+x_{m}\right)
$$

and obtain by (4.7)

$$
\sup _{0<t<1}\left\|N\left(v^{m}\right)(\cdot, t)\right\|_{\infty}=1
$$

and

$$
N\left(v^{m}\right)(0,1) \geq 1 / 2 \text {. }
$$

Furthermore, by (4.8)

$$
\left\|v_{0}^{m}\right\|_{B M O_{b}^{\mu_{m}, \nu_{m}}\left(\Omega_{m}\right)} \rightarrow 0 \quad(m \rightarrow \infty),
$$

where $\mu_{m}=\tau_{m}^{-1 / 2} \mu, \nu_{m}=\tau_{m}^{-1 / 2} \nu$ and

$$
\Omega_{m}:=\left\{x \in \mathbb{R}^{n}: x=\left(y-x_{m}\right) / \tau_{m}^{1 / 2}, y \in \Omega\right\} .
$$

Then $v^{m}$ solves the heat equation (4.1) in the rescaled domain $\Omega_{m}$.

Now let $c_{m}:=\operatorname{dist}\left(x_{m}, \partial \Omega\right) /\left(\tau_{m}^{1 / 2}\right)=\operatorname{dist}\left(0, \partial \Omega_{m}\right)$. We distinguish between the two cases $\lim \sup _{m \rightarrow \infty} c_{m}=\infty$ and $\limsup _{m \rightarrow \infty} c_{m}<\infty$. If $\limsup _{m \rightarrow \infty} c_{m}=\infty$ we can take a subsequence such that $\lim _{m \rightarrow \infty} c_{m}=\infty$. Then $\Omega_{m}$ expands to $\mathbb{R}^{n}$. Thus we obtain for every function $\varphi \in C_{c}^{\infty}\left(\mathbb{R}^{n} \times\right.$ $[0,1))$

$$
\int_{0}^{1} \int_{\mathbb{R}^{n}} v^{m}(x, t)\left(\Delta \varphi(x, t)+\varphi_{t}(x, t)\right) d x d t=-\int_{\mathbb{R}^{n}} v_{0}^{m}(x) \varphi(x, 0) d x
$$

and the same equality for the partial derivatives

$$
\int_{0}^{1} \int_{\mathbb{R}^{n}} \partial_{i} v^{m}(x, t)\left(\Delta \varphi(x, t)+\varphi_{t}(x, t)\right) d x d t=\int_{\mathbb{R}^{n}} v_{0}^{m}(x) \partial_{i} \varphi(x, 0) d x,
$$

where the right-hand side converges to zero by $(4.12)$ and $\int_{\mathbb{R}^{n}} \partial_{i} \varphi(x, 0) d x=$ 0. By local Hölder estimates (cf. [29, Chapter IV, Theorem 10.1]) we obtain that $v^{m}$ satisfies not only (4.10) but also Hölder estimates in the second derivative and time derivative. Therefore, we can obtain a subsequence 
again denoted by $v^{m}$ such that $\nabla v^{m}, \nabla^{2} v^{m}, v_{t}^{m}$ converge locally uniformly to some $g, \nabla g, h$. In the limit the equation (4.13) becomes

$$
\int_{0}^{1} \int_{\mathbb{R}^{n}} g\left(\Delta \varphi(x, t)+\varphi_{t}(x, t)\right) d x d t=0
$$

with $t^{1 / 2}\|g\|_{\infty} \leq c$ by (4.10). By the uniqueness result of Chung on the heat equation (cf. [8, Theorem 3.1 and Theorem 3.2]) we get that $g=0$. Then $\nabla g=0$ as well. By $\lim _{m \rightarrow \infty} \nabla^{2} v^{m}=\nabla g=0$ and $v_{t}^{m}=\Delta v^{m}$ we see that $h$ needs to vanish, too. We now have proved that $N\left(v^{m}\right)$ converges locally uniformly to 0 which is a contradiction to (4.11).

Now we have to consider the case $\limsup _{m \rightarrow \infty} c_{m}<\infty$. Then there is a subsequence satisfying $\lim _{m \rightarrow \infty} c_{m}=c_{0} \in[0, \infty)$. Then $\Omega_{m}$ expands to a half space $\mathbb{R}_{+,-c_{0}}^{n}:=\left\{x \in \mathbb{R}^{n}: x_{n}>-c_{0}\right\}$ (cf. [1] and [2]). Again, by local Hölder estimates we obtain that $v^{m}$ satisfies Hölder estimates in the second derivative and time derivative together with (4.10). Furthermore, by the boundary condition and (4.10) we can see that $v^{m}$ is locally bounded and we thus get that $v^{m}, \nabla v^{m}, \nabla^{2} v^{m}, v_{t}^{m}$ converge locally uniformly to some $v, \nabla v, \nabla^{2} v, v_{t}$. The limit $v$ then satisfies for all $\varphi \in C_{c}^{\infty}\left(\mathbb{R}_{+,-c_{0}}^{n} \times[0,1)\right)$ the equation

$$
\begin{aligned}
\int_{0}^{1} \int_{\mathbb{R}_{+,-c_{0}}^{n}} v(x, t)\left(\Delta \varphi(x, t)+\varphi_{t}(x, t)\right) d x d t & \\
= & =\lim _{m \rightarrow \infty} \int_{\mathbb{R}_{+,-c_{0}}^{n}} v_{0}^{m}(x, t) \varphi(x, 0) d x,
\end{aligned}
$$

where the right-hand side is equal to 0 by (4.12). Thus $v$ satisfies the homogeneous heat equation (4.1) in $\mathbb{R}_{+,-c_{0}}^{n}$. By (4.10) and the boundary condition we know that $v$ is bounded by $C t^{1 / 2}\left(x_{n}+c_{0}\right)$. If we take the odd extension $\bar{v}$ of $v$ to $\mathbb{R}^{n}$, the extension still satisfies the heat equation with initial data $\bar{v}_{0}=0$ and the estimate $\bar{v}(x, t) \leq C t^{1 / 2}\left(\left|x_{n}\right|+c_{0}\right)$. By the uniqueness result of Chung (cf. [8, Theorem 3.1 and Theorem 3.2]) we obtain that $v=0$. Thus $v$ and its derivatives converge locally uniformly to 0 which is again a contradiction to (4.10).

We have now proved that the statement holds for all $u_{0} \in C_{c}^{\infty}(\Omega)$. By density we can extend the estimate to $V M O_{b, 0}^{\mu, \nu}$.

We will now present the key steps for proving the boundedness of $\|u(t)\|_{B M O_{b}^{\mu, \nu}}$.

Lemma 9. Let $\Omega$ be a domain with uniformly $C^{3}$-boundary. Let $\mu, \nu \in$ $(0, \infty]$. Then there exist constants $C>0$ and $T_{0}>0$ such that for all $u_{0} \in V M O_{b, 0}^{\mu, \nu}(\Omega)$ there is a solution $u$ to (4.1) such that

(1) For all $x \in \Omega, r>0$ with $B_{r}(x) \subset \Omega$ and $t \in\left(0, T_{0}\right)$

$$
\left|u_{B_{r}(x)}(t)-u_{0 B_{r}(x)}\right| \leq C \frac{t^{\frac{1}{2}}}{r}\left\|u_{0}\right\|_{B M O_{b}^{\mu, \nu}}
$$

(2) For all $x \in \Omega, 0<r<\mu$ with $B_{r}(x) \subset \Omega$ and $t \in\left(0, T_{0}\right)$

$$
\frac{1}{\left|B_{r}(x)\right|} \int_{B_{r}(x)}\left|u(y, t)-u_{B_{r}(x)}(t)\right|^{2} d y \leq C\left(1+\frac{t}{r^{2}}\right)\left\|u_{0}\right\|_{B M O_{b}^{\mu, \nu}}^{2} .
$$


(3) For all $x \in \Omega, 0<r<\mu$ with $B_{r}(x) \subset \Omega$ and $t \in\left(0, T_{0}\right)$

$$
\frac{1}{\left|B_{r}(x)\right|} \int_{B_{r}(x)}\left|u(y, t)-u_{B_{r}(x)}(t)\right|^{2} d y \leq C \frac{r^{2}}{t}\left\|u_{0}\right\|_{B M O_{b}^{\mu, \nu}}^{2}
$$

(4) If $\nu \leq R^{*}$, then for all $x_{0} \in \partial \Omega, 0<r<\nu$ and $t \in\left(0, T_{0}\right)$

$$
\frac{1}{r^{n}} \int_{B_{r}\left(x_{0}\right) \cap \Omega}|u(y, t)|^{2} d y \leq C\left(\left\|u_{0}\right\|_{B M O_{b}^{\mu, \nu}}^{2}+\left[u_{0}\right]_{b^{\nu} 2}\right) .
$$

Proof. We will only give the key steps of the proof since the statements mainly follow from Theorem 17 and standard calculations. In Section 3 of [3] this argument has been carried out in detail for the Stokes equations and by ignoring the pressure term there one gets the result for the heat equation.

For proving (1) we use the equality $\int_{0}^{t} u_{s}(s) d s-u_{0}=u(t),(4.1)_{1}$, integration by parts and the estimate of Theorem 17 on $\nabla u$.

For proving (2) we again use the equality $\int_{0}^{t} u_{s}(s) d s-u_{0}=u(t),(4.1)_{1}$, integration by parts and the estimate of Theorem 17 on $\nabla u$ and combine it with the estimate of (1). The statement (3) follows directly from Poincaré's inequality.

In order to prove (4) we use again the equality $\int_{0}^{t} u_{s}(s) d s-u_{0}=u(t)$, $(4.1)_{1}$, integration by parts and the estimate of Theorem 17 on $\nabla u$. Compared to Theorem 3.4 in [3], where the estimate was proved for the Stokes equations and the smallness assumption on $\nu$ was also necessary for obtaining control on the constants that appear in estimating the pressure term, the assumption here is only necessary for ensuring that integration by parts is possible. Thus $\nu$ can be taken larger if for all $B_{r}\left(x_{0}\right) \cap \Omega$ with $x_{0} \in \partial \Omega$ and $r<\nu$ integration by parts is possible.

By the equivalence between $B M O_{b}^{\mu, \nu}$ and $B M O_{b}^{\mu, \nu} 2$ of Theorem 13 the following theorem follows.

Theorem 18. Let $\Omega$ be a domain with uniformly $C^{3}$-boundary. Let $\mu \in$ $(0, \infty], \nu \in\left(0, R^{*}\right]$. Let $\nu$ be finite if $\mu$ is finite. Then there exist constants $C>0$ and $T_{0}>0$ such that for all $u_{0} \in V M O_{b, 0}^{\mu, \nu}(\Omega)$ there is a solution $u$ to (4.1) satisfying

$$
\begin{array}{r}
\sup _{0<t<T_{0}}\left(\|u(t)\|_{B M O_{b}^{\mu, \nu}+t^{\frac{1}{2}}\|\nabla u(t)\|_{\infty}+t\left\|\nabla^{2} u(t)\right\|_{\infty}+} t\left\|u_{t}(t)\right\|_{\infty}\right) \\
\leq C\left\|u_{0}\right\|_{B M O_{b}^{\mu, \nu}}
\end{array}
$$

By Lemma 8 we can see that $u(t) \in W_{0}^{1, p}(\Omega) \subset V M O_{b, 0}^{\mu, \nu}(\Omega)$ with $p>n$ such that we can choose an arbitrary $T_{0} \in(0, \infty)$ by iteration and get the same estimate with a different constant $C_{T_{0}}$. In particular, the solution operator $H$ is a $C_{0}$ analytic semigroup in $V M O_{b, 0}^{\mu, \nu}(\Omega)$.

Remark 7. If one replaces $V M O_{b, 0}^{\mu, \nu}(\Omega)$ by $V M O_{b, 0}^{M}(\Omega)$ in the above theorem the statement still holds. 


\section{Applications to the Stokes semigroup}

In this section we will give some applications of the results for the Stokes semigroup which is the solution operator $S: u_{0} \mapsto S(t) u_{0}=u(t)$ of the equation

$$
\begin{array}{rll}
u_{t}-\Delta u+\nabla \pi & =0 & \text { in } \Omega \times(0, T), \\
\operatorname{div} u & =0 & \text { in } \Omega \times(0, T), \\
u & =0 & \text { on } \partial \Omega \times(0, T), \\
u(0) & =u_{0} . &
\end{array}
$$

It was proved in [3] that in bounded domains the Stokes semigroup is analytic in $V M O_{b, 0, \sigma}^{\mu, \nu}(\Omega)$ for sufficiently small $\nu$. By the equivalence result of Theorem 5 we can extend this result to general $\mu, \nu \in(0, \infty]$. We can further prove by the embedding theorem that the semigroup has the following property.

Theorem 19. Let $\Omega$ be a bounded $C^{3}$-domain, $\mu, \nu \in(0, \infty]$. Let $S$ be the Stokes semigroup on $\operatorname{VMO}_{b, 0, \sigma}^{\mu, \nu}(\Omega)$. Then $S$ is a bounded semigroup.

Proof. For every $T_{0} \in(0, \infty)$ there is some constant $C_{T_{0}}$ such that

$$
\sup _{0<t<T_{0}}\|u(t)\|_{B M O_{b}^{\mu, \nu}} \leq C_{T_{0}}\left\|u_{0}\right\|_{B M O_{b}^{\mu, \nu}}
$$

by [3, Theorem 3.5] and the equivalence result of Theorem 5 . Thus we can now assume $t \geq 1$. Let $p>n$. By the embedding of Theorem 12 we obtain that $u_{0} \in V M O_{b, 0, \sigma}^{\mu, \nu}(\Omega) \subset L_{\sigma}^{p}(\Omega)$. Let $u(t):=S(t) u_{0}$ be the solution to the homogeneous Stokes equations with initial data $u_{0}$. By $L^{p}$-theory (see e.g. [15]) we obtain for $t \geq 1$

$$
\begin{aligned}
\|u(t)\|_{1, p} & \leq\|u(t)\|_{p}+t^{1 / 2}\|\nabla u(t)\|_{p} \\
& \leq C\left\|u_{0}\right\|_{p} \\
& \leq C\left\|u_{0}\right\|_{B M O_{b}^{\mu, \nu} .}
\end{aligned}
$$

By the embedding $W^{1, p}(\Omega) \hookrightarrow L^{\infty}(\Omega) \hookrightarrow B M O_{b}^{\mu, \nu}(\Omega)$ we can conclude

$$
\|u(t)\|_{B M O_{b}^{\mu, \nu}} \leq C\left\|u_{0}\right\|_{B M O_{b}^{\mu, \nu}} .
$$

We can further extend the main result of [3] to the analyticity of the Stokes semigroup $S$ in $V M O_{b, 0, \sigma}^{M}$ in a suitable class of domains.

Theorem 20. Let $\Omega$ be a uniformly $C^{3}$-domain that is admissible in the sense of [1]. Then the solution operator $S$ to (5.1) is a $C_{0}$ analytic semigroup in $V M O_{b, 0, \sigma}^{M}(\Omega)$.

Proof. The proof can be copied almost verbatim from [3]. We will just give a short sketch on the main ideas and main differences. The gradient and time derivative estimate with respect to the $B M O_{b}^{M}$-norm have been proved in Remark 6.4 of [2]. For the estimate $\|u(t)\|_{B M O_{b}^{M}} \leq C\left\|u_{0}\right\|_{B M O_{b}^{M}}$ for $t<T_{0}$ we use similar ideas to those of Section 4 and [3], i.e., integrating by parts, fundamental theorem of calculus, using the gradient estimate and applying an equivalence result as well as an estimate for controlling the pressure term. The equivalence result we need to apply in this case is the statement 
of Theorem 14. The pressure estimate needs then only to be considered in balls since all subdomains of $\Omega$ appearing in the definition of the $B M O_{b}^{M}$ norm are balls. The constant in this estimate (cf. Theorem 2.1 in [3]) is scale invariant such that we have suitable control on the pressure term in every ball.

Remark 8. (1) This theorem avoids the previously necessary assumption to consider only small domains in the boundary seminorm, which in [3] was ensured by taking $\nu$ small.

(2) By Theorem 10 one obtains that if $\Omega$ is a Lipschitz half space that is admissible and uniformly $C^{3}$, then $S$ is an analytic semigroup in $V M O_{b, 0, \sigma}^{\infty, \infty}(\Omega)$. Except for the case of the half-space this result was not included in the main result of [3] since in all other cases $\nu$ needed to be finite.

(3) The analyticity in $V M O_{b, 0, \sigma}^{\infty, \infty}(\Omega)$ for a sector-like domain lying above a $C^{3}$-graph boundary as considered in [4] provides another approach to the proof of the $L^{p}$-analyticity for $p \in(2, \infty)$ in this domain. This was done there by interpolating $L^{2}-L^{2}$ estimates with $L^{\infty}-B M O_{b}^{\infty, \nu}$ estimates for $S(t) Q u_{0}$ and $\frac{\mathrm{d}}{\mathrm{d} t} S(t) Q u_{0}$. Here, $\nu$ could be chosen arbitrarily, $u_{0} \in C_{c}(\Omega)$ and $Q$ is a projection operator from $L^{2} \cap L^{\infty}$ onto $L_{\sigma}^{2} \cap V M O_{b, 0, \sigma}^{\infty, \nu}$. For the proof in [4] it was assumed that $\nu$ is sufficiently small. By using the analyticity result of Theorem 20 one can now also assume $\nu=\infty$ in the proof. Note that for some of these domains for sufficiently large $p$ the $L^{p}$-Helmholtz decomposition fails to hold.

\section{REFERENCES}

[1] Abe, K., Giga, Y.: Analyticity of the Stokes semigroup in spaces of bounded functions. Acta Math. 211, 1-46 (2013)

[2] Bolkart, M., Giga, Y.: On $L^{\infty}-B M O$ estimates for derivatives of the Stokes semigroup. Preprint. http://eprints3.math.sci.hokudai.ac.jp/2365/ Math. Z., to appear

[3] Bolkart, M., Giga, Y., Suzuki, T.: Analyticity of the Stokes semigroup in BMO-type spaces. Preprint. http://eprints3.math.sci.hokudai.ac.jp/2378/

[4] Bolkart, M., Giga, Y., Miura, T.H., Tsutsui, Y., Suzuki, T.: On analyticity of the $L^{p}$-Stokes semigroup for some non-Helmholtz domains. Preprint. http://eprints3.math.sci.hokudai.ac.jp/2382/

[5] Bony J.-M.: Calcul symbolique et propagation des singularités pour les équations aux dérivées partielles non linéaires, Ann. Sci. École Norm. Sup. 14 209-246 (1981)

[6] Carleson, L.: On convergence and growth of partial sums of Fourier series, Acta Math. 116, 135-157 (1966)

[7] Carpio, A.: Large-Time Behavior in Incompressible Navier-Stokes Equations. SIAM J. Math. Anal. 27, 449-475 (1996)

[8] Chung, S. Y.: Uniqueness in the Cauchy problem for the heat equation. Proc. Edinburgh Math. Soc. 42, 455-468 (1999)

[9] Coifman, R., Rochberg, R., Weiss, G.: Factorization theorems for Hardy spaces in several variables. Ann. of Math. 103, 611-635 (1976)

[10] David, G., Journé, J.-L.: A boundedness criterion for generalized Calderón-Zygmund operators, Ann. of Math. 120, 371-397 (1984)

[11] Fefferman, C.: Characterizations of bounded mean oscillation. Bull. Amer. Math. Soc. 77, 587-588 (1971)

[12] Fefferman, C., Stein E. M.: $H^{p}$ spaces of several variables. Acta Math. 129, 137-193 (1972) 
[13] Garcia-Cuerva, J., Rubio de Francia, J. L.: Weighted norm inequalities and related topics. North-Holland, Mathematics Studies, 116. Amsterdam-New York-Oxford, (1985)

[14] Gehring, F. W., Osgood, B. G.: Uniform domains and the quasihyperbolic metric. J. Analyse Math. 36, 50-74 (1979)

[15] Giga, Y.: Analyticity of the semigroup generated by the Stokes operator in $L^{r}$ spaces. Math. Z. 178, 297-329 (1981)

[16] Giga, Y., Matsui, S., Shimizu, Y.: On estimates in Hardy spaces for the Stokes flow in a half space. Math. Z. 231, 383-396 (1999)

[17] Gilbarg, D., Trudinger, N. S.: Elliptic Partial Differential Equations of Second Order. Grundlehren der Mathematischen Wissenschaften Vol. 224. Springer-Verlag, Berlin (1977)

[18] Gulisashvili, A.: On the Kato Classes of Distributions and the BMO-Classes. In: Differential Equations And Control Theory. Lecture Notes in Pure and Appl. Math. 225, pp. 159-176. Dekker, New York (2002)

[19] Hanks, R.: Interpolation by the Real Method between $B M O, L^{\alpha}(0<\alpha<\infty)$ and $H^{\alpha}(0<\alpha<\infty)$. Indiana Univ. Math. J. 26, 679-689 (1977)

[20] Hayashi, H., Ogawa, T.: $L^{p}-L^{q}$ type estimate for the fractional order Laplacian in the Hardy space and global existence of the dissipative quasi-geostrophic equation, Adv. Differ. Equ. Control Process. 5, 1-36 (2010)

[21] Janson, S., Jones, P. W.: Interpolation between $H^{p}$ spaces: the complex method, J. Funct. Anal. 48, 58-80 (1982)

[22] John, F., Nirenberg, L.: On functions of bounded mean oscillation. Comm. Pure Appl. Math. 14 415-426 (1961)

[23] Jones, P. W.: Extension Theorems for BMO. Indiana Univ. Math. J. 29, 41-66 (1980)

[24] Koch, H., Tataru D.: Well-posedness for the Navier-Stokes equations, Adv. Math. 157, 22-35 (2001)

[25] Kozono, H., Ogawa, T., Taniuchi, Y.: Navier-Stokes equations in the Besov space near $L^{\infty}$ and $B M O$, Kyushu J. Math. 57, 303-324 (2003)

[26] Kozono, H., Taniuchi, Y.: Bilinear estimates in BMO and the Navier-Stokes equations, Math. Z. 235, 173-194 (2000)

[27] Kozono, H., Wadade, H.: Remarks on Gagliardo-Nirenberg type inequality with critical Sobolev space and BMO. Math. Z. 259, 935-950 (2008)

[28] Krantz, S. G., Parks, H. R.: The implicit function theorem. History, theory, and applications. Birkhäuser Boston, Inc., Boston, MA (2002)

[29] Ladyženskaja, O. A., Solonnikov, V. A., Ural'ceva, N. N.: Linear and quasilinear equations of parabolic type. Translations of Mathematical Monographs Vol. 23, American Mathematical Society, Providence, R.I. (1968)

[30] Lunardi, A.: Analytic semigroups and optimal regularity in parabolic problems. Progress in Nonlinear Differential Equations and their Applications, 16. Birkhäuser Verlag, Basel (1995)

[31] Miyachi, A.: $H^{p}$ spaces over open subsets of $R^{n}$. Studia Math. 95, 205-228 (1990)

[32] Miyakawa, T.: Hardy spaces of solenoidal vector fields, with applications to the Navier-Stokes equations. Kyoushu J. Math. 50, 1-64 (1996)

[33] Miyakawa, T.: Application of the Hardy space techniques to time decay problem for incompressible Navier-Stokes flows in $\mathbb{R}^{n}$. Funkcial. Ekvac. 41, 383-434 (1998)

[34] Ogawa, T.; Real analytic methods of nonlinear evolution equations (in Japanese), Recent mathematics series of Springer 18, Maruzen Publishing, Tokyo 262-263 (2013)

[35] Staples, S. G.: $L^{p}$-averaging domains and the Poincaré inequality. Ann. Acad. Sci. Fenn. Ser. A I Math. 14, 103-127 (1989)

[36] Stein, E. M.: Singular integrals and differentiability properties of functions. Princeton Mathematical Series, No. 30. Princeton University Press, Princeton, N.J. (1970)

[37] Stein, E. M.: Harmonic Analysis, Princeton Mathematical Series, Vol. 43, Princeton University Press, Princeton (1993)

[38] Triebel, H.: Theory of Function Spaces, Monographs in Mathematics, 78. Birkhäuser Verlag, Basel (1983) 
[39] Uchiyama, A.: A constructive proof of the Fefferman-Stein decomposition of $B M O\left(\mathbb{R}^{n}\right)$, Acta Math. 148, 215-241 (1982)

Fachbereich Mathematik, Technische Universität Darmstadt, SchlossgartenStrasse 7, 64289 Darmstadt, Germany

E-mail address: bolkart@mathematik.tu-darmstadt.de

Graduate School of Mathematical Sciences, University of Tokyo, Komaba 3-8-1, Meguro-Ku, TOKYo 153-8914, JapAN

E-mail address: labgiga@ms.u-tokyo.ac.jp

Graduate School of Mathematical Sciences, University of Tokyo, Komaba 3-8-1, Meguro-Ku, Tokyo 153-8914, Japan

E-mail address: tsuzuki@ms.u-tokyo.ac.jp

Department of Mathematical Sciences, Faculty of Science, Shinshu UniverSity, Asahi 3-1-1, Matsumoto, Nagano, 390-8621, Japan

E-mail address: tsutsui@shinshu-u.ac.jp 